$8 \quad 2$ Lead contact

9 *Correspondence: aballay@ohsu.edu

\title{
Immunity-longevity tradeoff neurally controlled by GABAergic transcription factor PITX1/UNC-30
}

\author{
Benson Otarigho ${ }^{1}$ and Alejandro Aballay ${ }^{1,2, *}$
}
${ }^{1}$ Department of Molecular Microbiology \& Immunology, Oregon Health \& Science University, Portland, OR 97239, USA

\section{Abstract}

A body of evidence indicates that metazoan immune and aging pathways are largely interconnected, but the mechanisms involved in their homeostatic control remain unclear. In this

17 study, we found that the PITX (paired like homeodomain) transcription factor UNC-30 controls the tradeoff between immunity and longevity from the nervous system in Caenorhabditis elegans. PITX/UNC-30 functional loss enhanced immunity in a GATA/ELT-2- and p38 MAPK/PMK-1-dependent manner and reduced longevity by activating MXD/MDL-1 and the

22 stimulatory functions of PITX/UNC-30 required the sensory neuron ASG and a neurotransmitter

23 signaling pathway controlled by NPR-1, which is a G protein-coupled receptor related to

24 mammalian neuropeptide Y receptors. Our findings uncovered a suppressive role of GABAergic

25 signaling in the neural control of a biological tradeoff where energy is allocated towards

26 immunity at the expense of longevity.

Keywords: GABAergic, tradeoff, immunity, longevity, neurotransmitters, and neuropeptides

30

31 
Introduction

33 The activation of an immunological response is an essential and costly energetic physiological

34 process that results in the reallocation of resources, causing changes in the mechanisms that control general somatic maintenance (Ayres, 2020; Ganeshan and Chawla, 2014; Ganeshan et al., 2019; Jung et al., 2019). A tightly controlled immune activation is critical as its dysregulation has a major impact on a number of vital biological functions (Bird, 2019; Ganeshan et al., 2019; Jung et al., 2019; Kuchroo et al., 2012; Libert et al., 2006). In addition, the significant metabolic challenge posed by immune activation, immunopathology, and immune homeostasis necessitates an allocation of nutrients and energy that may lead to shortened longevity (Ayres, 2020; Backhed et al., 2019; Franceschi et al., 2018; Kaiser et al., 2020; Riera and Dillin, 2015; Solana et al., 2006; Tracey, 2002; Wani et al., 2019; Wu et al., 2019). Hence, the imbalance caused by immune activation results in a tradeoff between immunity and longevity that is evolutionarily conserved throughout the animal kingdom.

While the aforementioned changes to maintain homeostasis suggest the existence of a tradeoff between immunity and longevity across metazoans, the specific pathways involved in those tradeoffs and the underlying mechanisms that control them remain unknown. Several conserved pathways, including the DAF-2 (homolog of insulin-like growth factor 1, IGF1), AGE-1 (homolog of phosphatidylinositol 3-kinase, PI3K), DAF-16 (homolog of FOXO transcription factor), and HFS-1 (homolog of heat shock transcription factor 1, HSF1) pathways, control both immunity and longevity in the nematode Caenorhabditis elegans (Chávez et al.,

52 2007; Garsin et al., 2003; Hajdu-Cronin et al., 2004; Libina et al., 2003; Mohri-Shiomi and

53 Garsin, 2008; Morris et al., 1996; Murphy et al., 2003; Singh and Aballay, 2006). However,

54 whether and how these interconnected immune and longevity pathways are regulated by tradeoff

55 mechanisms still needs to be determined. The nervous system, which can integrate different cues

56 in milliseconds and interpret conflicting stimuli, is well-equipped to effectively regulate

57 tradeoffs.

To investigate the role of the nervous system in the control of the immunity-longevity tradeoff, we studied the GABAergic transcription factor PITX/UNC-30 in C. elegans. PITX/UNC-30 transcriptionally regulates the expression of GAD/UNC-25 and SLC32A1/UNC-

6147 that are critical in the biosynthesis, packaging, and trafficking of GABA (Cinar et al., 2005; 62 Eastman et al., 1999b; Garcia et al., 2007; Gendrel et al., 2016; McIntire et al., 1997; Mclntire et 
al., 1993a; Mclntire et al., 1993b). We found that UNC-30 loss-of-function mutations led to enhanced resistance to killing by Gram-negative and Gram-positive bacterial pathogens. pathogen infection was mediated by the GATA transcription factor ELT-2 and the p38 mitogenactivated protein kinase PMK-1. The reduced longevity phenotype of UNC-30 mutants was due to higher activity of the MAX dimerization protein MXD/MDL-1 and the C2H2-type zinc finger transcription factor PQM-1. We further found that neuropeptide signaling mediated by the NPYR/NPR-1 pathway acts downstream of UNC-30 and the amphid sensory neuron ASG to control the tradeoff. Our findings highlight a neuronal network that controls the tradeoff between

72 immunity and longevity. Given the conservation of GABAergic signaling and the longevity and

73 immune pathways involved in the tradeoff, our findings raise the possibility that a similar process may occur in higher metazoans, including humans.

\section{Results}

\section{Neuronal UNC-30 controls the immunity-longevity tradeoff}

As a first step to understand the role of UNC-30 in the control of the immunity-longevity tradeoff, we studied the survival of $u n c-30$ loss-of-function animals infected with the human opportunistic pathogen $P$. aeruginosa strain PA14. We found that unc-30(ok613) mutants exhibited enhanced survival against $P$. aeruginosa-mediated killing compared to wild-type animals (Figure 1A). We also found that the unc-30(ok613) mutants exhibited less visible bacterial colonization and significantly reduced colony-forming units compared to wild-type animals (Figure 1B-C). Different $u n c-30$ strains also displayed enhanced survival against $P$. aeruginosa compared to wild-type animals (Figure S1A). When exposed to E. coli, which is the food source of $C$. elegans in the laboratory, unc-30(ok613) mutants exhibited enhanced survival compared to wild-type animals (Figure 1D and S1B). Because proliferating live E. coli is a cause of death in C. elegans and animals deficient in the immune response are persistently colonized and killed by the bacterium (Garigan et al., 2002; Otarigho and Aballay, 2020; Sutphin and Kaeberlein, 2009), we first investigated the survival of unc-30(ok613) animals on killed E. coli.

91 Because animals fed heat-killed E. coli exhibit bloated intestinal lumens that result in up-

92 regulation of immune pathways (Singh and Aballay, 2019b), we exposed the animals to lawns of 93 E. coli killed by ultraviolet light (UV) that does not cause any intestinal distension (S2A-J). As 
shown in Figure 1E, the longevity of unc-30(ok613) animals is shorter than that of wild-type animals when exposed to UV-killed $E$. coli. As ampicillin was added to prevent the growth of any bacteria that might have escaped the heat treatment, we studied whether the addition of the antibiotic would impact the lifespan of the animals. As shown in Figure S1B, ampicillin did not affect the lifespan of unc-30(ok613) mutants.

UNC-30 is mainly expressed in three sensory neuronal cells (ASG, PVD, and OLL), three interneurons (PVP, RID, and RIH), and two motor neurons (DD and VD), as well as in the intestine (Cinar et al., 2005; Eastman et al., 1999b; Jin et al., 1994; Kurup and Jin, 2016; Walton et al., 2015; Westmoreland et al., 2001). To study whether UNC-30 may act cell autonomously in the intestine to control immune pathways, we knocked down UNC-30 in the intestine of an

104 RNAi intestine-specific strain, MGH171. The lack of a significant difference between control and $u n c-30$ RNAi animals (Figure $1 \mathrm{~F}$ ) suggests that $u n c-30$ does not function in the intestine to control immunity and that it may control the immunity-longevity tradeoff from the nervous system. To study whether UNC-30 controls the immunity-longevity tradeoff in a cell nonautonomous manner from the nervous system, we rescued unc-30 under the control of a panneuronal promoter and exposed the animals to $P$. aeruginosa. As a control, unc-30 was also expressed using its native promoter. The results obtained show that UNC-30 expression driven by its own or the pan-neuronal promoter rescued both the enhanced survival of unc-30(ok613)

112 animals to $P$. aeruginosa-mediated killing (Figure $1 \mathrm{G}$ ) and the reduced longevity of the animals 113 grown on heat-killed E. coli (Figure 1E). To study the specificity of pathogen susceptibility of unc-30(ok613) animals, we infected them with other human bacterial pathogens, including the

115 Gram-negative bacterium Salmonella enterica strain 1344 and the Gram-positive bacteria

116 Staphylococcus aureus strain NCTCB325. Loss of UNC-30 enhanced the nematode's survival 117 against all bacterial pathogens studied (Figure 1H-I), suggesting that UNC-30 suppresses $C$. elegans general defense against bacterial pathogens. Because $C$. elegans naturally exhibits an

119 avoidance behavior when exposed to $P$. aeruginosa, which can be observed in conditions in 120 which the animals can freely enter and exit the bacterial lawn, we used plates that were 121 completely covered by bacteria to control for avoidance (full-lawn). The survival of unc-

$12230($ ok613) animals was also significantly higher than that of wild-type and unc-30 rescued 123 animals (Figure 1J). We also observed similar resistance to pathogen-mediated killing when the animals were exposed to full-lawns of S. enterica or S. aureus (Figure S3A-B). The neural 
expression of unc-30 rescued the survival defect of unc-30(ok613) animals (Figures 1G-J).

126 Consistent with the idea that the resistance of $u n c-30($ ok613) animals is not due to enhanced

127 pathogen avoidance, we found that the lawn occupancy of unc-30(ok613) mutants is comparable

128 to that of wild-type animals (Figure S3C).

129 C. elegans feeds on bacteria and the pharyngeal contraction/pumping is a direct measure of food intake (Cao et al., 2017; Sellegounder et al., 2019; Singh and Aballay, 2019c; Styer et al., 2008). Thus, we asked whether the resistance to infections and reduction of the lifespan of unc30(ok613) animals could be due to a reduction in pathogen intake. We, therefore, measured the pharyngeal pumping rates of $u n c-30($ ok613) and wild-type animals on P. aeruginosa bacterial lawns as well as on heat-killed E. coli. We found that $u n c-30($ ok613) animals exhibited pumping rates comparable to that of wild-type animals (Figure S3D), indicating that the dose of pathogens is similar in both cases. It has recently been demonstrated that bacterial accumulation in animals defective in the defecation motor program (DMP) causes intestinal distension that elicits a robust immune response (Singh and Aballay, 2019c) and modulates longevity (Kumar et al., 2019). We found that the defecation cycle of $u n c-30(0 k 613)$ animals is indistinguishable from that of wildtype animals (Figure S3E). Certain gene mutations in C. elegans affect the reproductive system, which could lead to reduced progeny or cause total sterility, resulting in increased resistance to pathogen infection (Berman and Kenyon, 2006; Powell and Ausubel, 2008). In addition, the tradeoff between immunity and longevity can be dependent on the reproductive dynamics of some mutant animals (Amrit et al., 2019). Thus, we compared the brood size of unc-30(ok613) and wild-type animals and did not observe any difference in the numbers of neither laid eggs nor brood size (Figure S4A-B). Taken together, these results suggest that $u n c-30$ functions in the nervous system to control a tradeoff between immunity and longevity.

UNC-30 inhibits the expression of immune and age-related genes

To dissect the UNC-30-dependent immune and longevity mechanisms involved in the control of

151 defense against pathogen exposure and longevity, we employed transcriptomic analyses to

152 identify dysregulated immune genes and pathways in uninfected and $P$. aeruginosa-infected unc153 30(ok613) and wild-type animals (Table S1). To identify gene groups that were controlled by 154 UNC-30, we performed an unbiased gene enrichment analysis using a Wormbase enrichment analysis tool, https://wormbase.org/tools/enrichment/tea/tea.cgi, (Angeles-Albores et al., 2016), 
that is specific for $C$. elegans gene data analyses. The 10 gene ontology clusters with the highest enrichment score of vital biological functions for upregulated and downregulated genes in both non-infected and infected groups are shown in Figure 2A and S5A-B. Overall, the gene expression data showed that the most enriched and highly significant upregulated genes were a part of neuropeptide signaling and immune/defense response pathways in both non-infected and infected groups (Figure 2A and Table S2). Other genes linked to biological processes such as metabolism, response to biotic stimuli, and IRE1-mediated unfolded protein response, were also upregulated in unc-30(ok613) animals. Consistently with the role of UNC-30 in the control of longevity (Figure 1E), genes involved in aging were also significantly enriched (Figure 2A).

To identify the potential immune pathways that may play a role in the pathogen resistance to the UNC-30-deficient animals, we performed a gene enrichment analysis using the immune gene subset by employing the Worm Exp tool (https://wormexp.zoologie.unikiel.de/wormexp/) (Yang et al., 2016), which integrates all published expression datasets for $C$. elegans to analyze and generate pathways. We found that pathways required for C. elegans defense against bacterial infections were highly enriched, including the ELT-2, PMK-1, PQM-1, and DAF-2/DAF-16 insulin pathways (Figure 2B and Table S3) (Aballay et al., 2003; Garsin et

172 al., 2003; Head et al., 2017; Huffman et al., 2004; Kerry et al., 2006; Kim et al., 2002; Murphy et al., 2003; Rajan et al., 2019; Shapira et al., 2006; Singh and Aballay, 2006, 2009; Troemel et al., 2006). We also noticed that several of the PMK-1 and DAF-16-dependent genes were also controlled by ELT-2 (Figure S6 and Tables S3), indicating that ELT-2-dependent genes may play a major role in the enhanced resistance to pathogen phenotype of unc-30(ok613) animals. Thus, we confirmed the role of neural UNC-30 in the control of ELT-2-dependent immune genes, including lys-1, K12H4.7, lys-4, lys-7, and lys-8 (Figure 2C). These results indicate that UNC-30 regulates $C$. elegans defense against bacterial infections mainly by activating immune genes, several of which are controlled by the ELT-2 immune transcription factor.

We further employed the Worm Exp tool (https://wormexp.zoologie.uni-

kiel.de/wormexp/) (Yang et al., 2016) to analyze the age determination genes to identify specific pathways that could be transcriptionally regulated by UNC-30. We found that the SKN-1, MDL-

185 (Riesen et al., 2014; Tepper et al., 2013a; Tepper et al., 2014; Tullet et al., 2008). Although the 
187

188

189

190

191

192

193

194

195

196

197

198

199

200

201

202

203

204

205

206

207

208

209

210

211

212

213

214

215

216

217

in unc-30(ok613) animals were under the control of the MDL-1 pathway (Figure 2E). Because the evolutionarily conserved transcription factors, MDL-1 and PQM-1 are known to suppress longevity and their inactivation results in lifespan extension (Riesen et al., 2014; Tepper et al., 2013b; Tepper et al., 2014), the upregulation of MDL-1 and PQM-1-dependent genes in unc30(ok613) animals could explain the reduced longevity of the animals. We confirmed the role of UNC-30 in the control of MDL-1 and PQM-1-dependent genes (Figure 2F). In summary, the transcriptomic analyses revealed that while neural expression of UNC-30 downregulates the expression of immune genes controlled by ELT-2 and PMK-1, it upregulates age-related genes controlled by MDL-1 and PQM-1, indicating that UNC-30 controls a tradeoff between immunity and longevity.

\section{UNC-30 functional loss enhances immunity via ELT-2 and PMK-1 and reduces longevity} via MDL-1 and PQM-1

To test the hypothesis that the enhanced resistance to $P$. aeruginosa infection of unc-30(ok613) animals is due to the upregulation of immune genes, we studied the role of suppression by RNAi of the immune pathways shown in Figure 2B. We inactivated elt-2, pqm-1, pmk-1, and daf-16 in WT and unc-30(ok613) animals and exposed them to P. aeruginosa. Unlike daf-16 and pqm-1 RNAi (Figure 3A-B), elt-2 RNAi was able to completely suppress the enhanced resistance to $P$. aeruginosa infection in unc-30(ok613) animals (Figure 3C). We also observed partial suppression of the enhanced pathogen resistance of unc-30(ok613) animals by pmk-1 RNAi (Figure 3D). The lack of suppression of the phenotype of $u n c-30($ ok613) animals by RNAi inhibition of daf-16 and pqm-1 was confirmed using daf-16 and pqm-1 mutant animals (Figure 3E-F). We also confirmed the partial suppression of the enhanced resistance to pathogen of unc30(ok613) animals by inhibition of the PMK-1 pathway using nsy-1(ag3) and sek-1(km4) animals, which carry mutations in the genes encoding two kinases that function upstream PMK-1 (Figure 3G-H). These results indicate that loss of UNC-30 enhances immunity against infection by increasing the activity of ELT-2 and the PMK-1 pathway.

The Venn diagram shown in Figure 4A indicates a poor overlap between aging and immune genes, suggesting that the misregulation of different pathways causes the immune and longevity phenotypes of animals deficient in UNC-30. Thus, we reasoned that the upregulation of the age-related genes in un-infected unc-30(ok613) mutants compared with wild-type animals 
218 (Figure 2A) could be responsible for the reduced longevity of unc-30(ok613) animals. Consistent 219 with this idea, a number of the age-related genes that are upregulated in unc-30(ok613) animals 220 have been reported to extend lifespan when mutated or inactivated by RNAi (Figure 4B and 221 Table S4). Moreover, the majority of age-related genes upregulated in unc-30(ok613) mutants 222 compared with wild-type animals are controlled by MDL-1 and PQM-1 (Figure 2E), which are 223 known to suppress longevity. Thus, we studied whether inactivation of MDL-1 or PQM-1 could 224 suppress the decreased longevity of unc-30(ok613) animals. As shown in Figures 4C and 4D, $225 \mathrm{mdl}-1(\mathrm{tm} 311)$ and pqm-1(ok485) mutation suppressed the reduced lifespan of unc-30(ok613) 226 animals. Consistent with the idea that higher activity of both MDL-1 and PQM-1 is responsible 227 for the reduced lifespan of unc-30(ok613) animals, the lifespan of the double mutants $m d l$ 228 1(tm311);unc-30(ok613) and pqm-1(ok485);unc-30(ok613) is shorter than that of the single 229 mutants $m d l-1(t m 311)$ and pqm-1(ok485), respectively (Figures 4C and 4D). To study whether 230 the longevity of the single mutants is greater than that of the double mutants due to higher PQM2311 or MDL-1 activity, we inhibited both PQM-1 and MDL-1 in unc-30(ok613) animals. As shown 232 in Figure 4E, the longevity of $m d l-1$ RNAi animals is not significantly different than that of pqm233 1(ok485);unc-30(ok613);mdl-1 RNAi animals. Inhibition of SKN-1 by RNAi reduced the 234 longevity of wild-type animals but had no effect on unc-30(ok613) mutants (Figure 4F). This is 235 expected because, unlike MDL-1 and PQM-1 that suppress longevity, SKN-1 promotes 236 longevity. Taken together, these results indicate that neuronal UNC-30 suppresses ELT-2- and 237 PMK-1 mediated immunity while prolonging longevity through the MDL-1 and PQM-1 238 pathways.

\section{Neuronal UNC-30 regulates the immunity-longevity tradeoff via neuropeptide signaling} The subset of misregulated genes most significantly enriched in either infected or uninfected

243 To identify potential neurotransmitter pathways that may be involved in the control of the 244 tradeoff between immunity and longevity, we performed gene enrichment analysis using the neuropeptide genes subset (Table S5). Our findings show that most of the neuropeptide genes are transcriptionally controlled by NPR-1 (Figure 5A and Table S5). NPR-1 is a G protein-coupled

247 receptor similar to neuropeptide Y receptor (NPYR) that controls C. elegans immune response 
2019c; Styer et al., 2008). Other genes are controlled by miRNA and LIN-28 (Figure 5A and in $u n c-30($ ok613) mutants compared to wild-type animals. We also found that the pan-neuronal expression of $u n c-30($ ok613) rescued the changes in expression of NPR-1-dependent neuropeptide genes (Figure 5B.

Because most unc-30(ok613) upregulated neuropeptide genes are NPYR/NPR-1dependent, we asked whether NPR-1 may be part of the UNC-30 pathway that controls the

256 immunity-longevity tradeoff. We hypothesized that if $n p r-1$ acts downstream of $u n c-30, n p r-1$

257 mutation should suppress the enhanced immunity and reduced longevity of unc-30(ok613) animals. Indeed, we found that the npr-1(ad609) mutation was able to suppress the immunity and longevity phenotypes of unc-30(ok613) animals (Figure 5C, 5D, and S7). We further studied NPR-1-regulated genes and found that, in addition to controlling the immune PMK-1 pathway (Styer et al., 2008), NPR-1 controls immune genes that are ELT-2 and PQM-1-dependent (Figure 5E and Table S6). Consistent with the suppression of the reduced longevity of unc-30(ok613) animals by mutation in $n p r-1$, we found that the $n p r-1($ ad609) mutation suppressed the missregulation of age-related genes observed in unc-30(ok613) animals (Figure 5F).

\section{GABAergic ASG neurons control the UNC-30-modulated immunity-longevity tradeoff}

267 Next, we asked which UNC-30-expressing neuronal cells could be involved in the control of the immune-longevity tradeoff. As a first step, we employed a two-component system to kill cells by using a reconstituted caspase (Chelur and Chalfie, 2007) to generate a PVP neuron ablated strain and that was exposed to $P$. aeruginosa. We focused on PVP because it is well connected to other

272 difference between the PVP(-) and wild-type animals (Figure 6A), suggesting that the

273 immunomodulatory function of UNC-30 is not linked to PVP neurons. However, we cannot rule 274 out the role of DD, VD, and VC in immunity.

275 We next studied whether other UNC-30-expressing cells (ASG, PVD, or OLL sensory

276 neurons) could control the defense response against pathogen infection. We employed an

277 interactive tissue-gene expression prediction tool (http://worm.princeton.edu) to predict the

278 expression enrichment level across different cells and tissues. The results obtained revealed that unc-30 is highly expressed in ASG, which is an amphid neuron (Figure S8B) (Li and Kim, 2008; 
Pereira et al., 2015; Pocock and Hobert, 2010). Also, we noticed that some of the neuropeptide genes, including flp-6, flp-13, flp-22, and ins-1, that are upregulated in unc-30(ok613) animals are expressed in ASG neurons (Li and Kim, 2008; Pereira et al., 2015; Pocock and Hobert, 2010). Thus, we studied the effect of ASG ablation in the control of defense against $P$. aeruginosa infection in wild-type and unc-30(ok613) animals. We found that ASG(-) animals exhibited resistance to $P$. aeruginosa-mediated killing similar to that of unc-30(ok613) animals (Figure 6B). The resistance to P. aeruginosa infection of unc-30(ok613) and ASG(-)::unc30(ok613) animals was also indistinguishable (Figure 6B). In addition, expression of unc-30 only in ASG neurons completely suppressed the pathogen resistance of unc-30(ok613) animals (Figure 6C), suggesting that the GABAergic ASG neuron plays a key role in the UNC-30 modulation of immunity. To further confirm the role of ASG neurons in the control of immunity, we quantified the expression of immune genes and found that they were upregulated in ASG(-) animals (Figure 6D).

We also investigated the lifespan of animals lacking ASG(-) or PVP(-). As shown in Figure 6E, ASG(-) but not PVP(-) animals exhibited a longer lifespan than control animals. We also found that ASG ablation partially rescued the short lifespan of unc-30(ok613) animals, suggesting that ASG neurons participate in the control of longevity by UNC-30 (Figure 6F). We also found that unc-30 expression in ASG neurons rescued the short lifespan of unc-30(ok613) animals (Figure 6G). In summary, our results suggest that neuronal UNC-30 and neuropeptide signaling regulate the tradeoff between immunity and longevity controlling the ELT-2 and PMK1 immune pathway and the MDL-1 and PQM-1 aging pathways (Figure 7).

\section{Discussion}

303 Biological tradeoffs are widely employed by metazoans to maximize the allocation of precious resources that are critical for organismal homeostasis. However, the mechanisms that control them at the whole animal level remain obscure. The nervous system provides a perfect partner to

306 help fine-tune tradeoffs because it can rapidly respond to many types of stimuli and is adept at 307 controlling opposite functions. (Eastman et al., 1999a; Jin et al., 1994; Westmoreland et al., 2001). Here, we show that UNC-30 functions in the nervous system from where, together with neuropeptide signaling, it can control the tradeoff between immunity and longevity. The absence 
3111 and PQM-1 and immune genes controlled by the ELT-2 and PMK-1. Furthermore, the

312 GABAergic neuron ASG and the NPR-1 neuropeptide signaling are part of the UNC-30 pathway

313 that controls the balance between immune activation and longevity.

314 Previous studies linked neurotransmitters such as dopamine and octopamine to the

315 control of immunity via the PMK-1 pathway (Cao and Aballay, 2016; Pinoli et al., 2017;

316 Sellegounder et al., 2019; Sun et al., 2011) and the X-box binding protein 1 (XBP-1) branch of

317 the canonical UPR pathway (Cao et al., 2017). Acetylcholine has also been connected to the

318 control of immunity via the intestinal epithelial Wnt Signaling (Labed et al., 2018). Furthermore,

319 serotonin was also found to modulate the immune response via G-protein GOA-1 (Gao) in rectal

320 epithelial cells (Anderson et al., 2013; Hoffman and Aballay, 2019). Unlike other

321 neurotransmitter pathways, the GABAergic signaling pathway modulates the immune response

322 to pathogens via the control of the expression of immune genes regulated by ELT-2 and PMK-1.

323 The UNC-30 pathway also controls genes that are involved in aging (Alcedo and Kenyon, 2004;

324 Apfeld and Kenyon, 1999; Jeong et al., 2012; Kenyon, 2010; Wolkow et al., 2000). The G

325 protein-coupled GABA receptor GBB-1 modulates longevity through G protein-PLCb, which

326 transmits longevity signals to FOXO/DAF-16 (Chun et al., 2015; Yuan et al., 2019). Our studies

327 highlight a role for neural UNC-30 in the integration of signals that control the balance between

328 the activation of both immune and longevity pathways that have opposite effects. While UNC-30

329 suppresses immunity by inhibiting the expression of ELT-2- and PMK-1-dependent genes, it

330 enhances longevity by inhibiting the activity of MDL-1 and PQM-1. Functional loss of MDL-1

331 and PQM-1 extends lifespan in C. elegans via DAF-2 insulin/IGF-1 (Johnson et al., 2014;

332 Nakamura et al., 2016; Riesen et al., 2014; Templeman et al., 2018; Tepper et al., 2013b; Tepper

333 et al., 2014). In addition, PQM-1 functions together with DAF-16 as a key transcriptional

334 regulator of DAF-2-mediated longevity (Tepper et al., 2013b). However, the lack of significant

335 enrichment in DAF-16-controled genes regulated by UNC-30 suggests that PQM-1 may also

336 control longevity in a DAF-16-independent manner.

337 Our findings suggest that ASG neurons play a key role in controlling the tradeoff

338 between immunity and longevity. They also express neuropeptides flp-6, flp-13, flp-22, and ins-1

339 (Li and Kim, 2008; Pereira et al., 2015; Pocock and Hobert, 2010), which we found are

340 upregulated in $u n c-30($ ok613) animals. Furthermore, ASG cells respond to neuropeptides $f l p-18$

341 and flp-21 via npr-1 (Bargmann, 2006; Li and Kim, 2008; Pereira et al., 2015; Pocock and 
Hobert, 2010; Rex et al., 2005; Sugiura et al., 2005), which were upregulated in unc-30(ok613) animals. These results argue strongly that ASG neurons are critical in the control of the longevity-immunity tradeoff. Both PMK-1 and ELT-2 function in the intestine to control immunity (Bolz et al., 2010; Head et al., 2017; Kerry et al., 2006; Kim et al., 2002; Shapira et al., 2006). In addition, the role of PQM-1 and MDL-1 in longevity has been linked to the intestine (Riesen et al., 2014; Tepper et al., 2013b), suggesting that UNC-30 controls the immunitylongevity tradeoff in a cell non-autonomous manner. A recent example of a tradeoff mechanism indicates that dietary restriction extends lifespan by downregulating the PMK-1-mediated immune signaling (Wu et al., 2019). Further work will be required to establish whether this and other tradeoffs are controlled cell non-autonomously by the nervous system.

Our results provide evidence that specific genes and neurons in the nervous system act in a cell non-autonomous manner to coordinately control immune and aging pathways. Organisms widely rely on tradeoff mechanisms when a given trait cannot increase without decreasing another to maintain homeostasis. Given the evolutionary conservation of UNC-30 and the immune and aging pathways that are neutrally controlled, the identification and characterization of the cues that the nervous system uses to control the immunity-longevity tradeoff in C. elegans should yield insights into similar mechanisms used across metazoans.

\section{Materials and Methods}

\section{Bacterial strains}

The bacterial strains used in this study are Escherichia coli OP50, E. coli HT115(DE3) (Brenner, 1974), Pseudomonas aeruginosa PA14, P. aeruginosa PA14-GFP (Tan et al., 1999), Salmonella enterica Serovar Typhimurium 1344 (Wray and Sojka, 1978), and Staphylococcus aureus strain NCTCB325 (Sifri et al., 2003). Gram-negative bacteria were grown in Luria-Bertani (LB) broth.

\section{C. elegans Strains and Growth Conditions}

371 Hermaphrodite C. elegans (var. Bristol) wild type (N2) was used as control unless otherwise

372 indicated. C. elegans strains CF1038 daf-16(mu86), KU25 pmk-1(km25), MGH171 alxIs9 [vha- 
CB318 unc-30(e318), CB845 unc-30(e191), SD39 unc-30(e596), GR2245 skn-1(mg570), RB711 pqm-1(ok485), KU4 sek-1(km4) and AU3 nsy-1(ag3) were obtained from the Caenorhabditis Genetics Center (University of Minnesota, Minneapolis, MN). $m d l-1(\operatorname{tm} 311)$ was obtained from National Bioresource Project (NBRP), Japan. npr-1(ad609);unc-30(ok613), pqm-1(ok485);unc30(ok613), daf-16(mu86); unc-30(ok613), sek-1(km4);unc-30(ok613), nsy-1;unc-30(ok613), mdl1(tm311);unc-30(ok613), and ASG(-)::unc-30(ok613) were obtained by standard genetic crosses. Rescued strain unc-30(ok613);Punc-30::unc-30, neuronal rescued strain unc-30(ok613);Prab3::unc-30 and unc-30 rescued in ASG neuron-specific, unc-30(ok613);Pgcy-15::unc-30 were generated as described below. The ASG(-) ablated Ex[Ptax-2::CZ::ced-3(p17)::unc-54 3'UTR + Plim-6::ced-3(p15)-NZ::unc-54 3'UTR, pRF4] and PVP(-) ablated Ex[Podr-2b::CZ::ced3(p17)::unc-54 3'UTR, Punc-53::ced-3(p15)-NZ::unc-54 3'UTR, pRF4] were also generated as described below. The strains were crossed with the wild-type laboratory N2.

All strains were grown at $20^{\circ} \mathrm{C}$ on nematode growth medium (NGM) plates seeded with E. coli OP50 as the food source (Brenner, 1974) unless otherwise indicated. The recipe for the control NGM plates is: $3 \mathrm{~g} / 1 \mathrm{NaCl}, 3 \mathrm{~g} / 1$ peptone, $20 \mathrm{~g} / \mathrm{l}$ agar, $5 \mu \mathrm{g} / \mathrm{ml}$ cholesterol, $1 \mathrm{mM} \mathrm{MgSO} 4$, $1 \mathrm{mM} \mathrm{CaCl} 2$, and $25 \mathrm{mM}$ potassium phosphate buffer ( $\mathrm{pH}$ 6.0). The NGM plates were without antibiotics except indicated.

\section{RNA Interference (RNAi)}

Knockdown of targeted genes was obtained using RNAi by feeding the animal with $E$. coli strain HT115(DE3) expressing double-stranded RNA (dsRNA) homologous to a target gene (Fraser et al., 2000; Timmons and Fire, 1998). RNAi was carried out as described previously (Sun et al., 2011; Singh and Aballay, 2017 ). Briefly, E. coli with the appropriate vectors were grown in LB broth containing ampicillin $(100 \mu \mathrm{g} / \mathrm{mL})$ and tetracycline $(12.5 \mu \mathrm{g} / \mathrm{mL})$ at $37^{\circ} \mathrm{C}$ overnight and plated onto NGM plates containing $100 \mu \mathrm{g} / \mathrm{mL}$ ampicillin and $3 \mathrm{mM}$ isopropyl $\beta$-Dthiogalactoside (IPTG) (RNAi plates). RNAi-expressing bacteria were grown at $37^{\circ} \mathrm{C}$ for $12-14$

400 hours. Gravid adults were transferred to RNAi-expressing bacterial lawns and allowed to lay 401 eggs for 2-3 hours. The gravid adults were removed, and the eggs were allowed to develop at $20^{\circ} \mathrm{C}$ to young adults. This was repeated for another generation (except for ELT-2 RNAi) before

403 the animals were used in the experiments. The RNAi clones were from the Ahringer RNAi 404 library. 


\section{C. elegans Survival Assay on Bacterial Pathogens}

$407 P$. aeruginosa and S. enterica were incubated in LB medium. S. aureus was incubated in TSA 408 medium with nalidixic acid $(10 \mu \mathrm{g} / \mathrm{mL})$. The incubations were done at $37^{\circ} \mathrm{C}$ with gentle shaking 409 for 12 hours. P. aeruginosa and S. enterica were grown on modified NGM agar medium $(0.35 \%$ 410 peptone). For partial lawn assays, $20 \mu \mathrm{l}$ of the overnight bacterial cultures were seeded at the 411 center of the relevant agar plates without spreading. For full lawn experiments, $20 \mu \mathrm{l}$ of the 412 bacterial culture were seeded and spread all over the surface of the agar plate. No antibiotic was 413 used for P. aeruginosa and S. enterica, while nalidixic acid $(10 \mu \mathrm{g} / \mathrm{mL})$ was used for the TSA

414 plates for S. aureus. The seeded plates were allowed to grow for 12 at $37^{\circ} \mathrm{C}$. The plates were left 415 at room temperature for at least $1 \mathrm{hr}$ before the infection experiments. 20 synchronized young 416 adult worms were transferred to the plates for infection, and three technical replicate plates were 417 set up for each condition ( $\mathrm{n}=60$ animals) and the experiments were performed in triplicate 418 (Table 7). The plates were then incubated at $25^{\circ} \mathrm{C}$. Scoring was performed every $12 \mathrm{hr}$ for $P$. aeruginosa and S. aureus, while $24 \mathrm{hr}$ for S. enterica. Worms were scored as dead if the animals 420 did not respond to touch by a worm pick or lack of pharyngeal pumping. Live animals were 421 transferred to fresh pathogen lawns each day. All C. elegans killing assays were performed three 422 times independently.

\section{Bacterial lawn avoidance assay}

425 Bacterial lawn avoidance assays were performed by $20 \mathrm{~mL}$ of $P$. aeruginosa PA14 on 3.5-cm 426 modified NGM agar $(0.35 \%$ peptone $)$ plates and cultured at $37^{\circ} \mathrm{C}$ overnight to have a partial 427 lawn. The modified NGM plates were left to cool to room temperature for about 1 hour, twenty

428 young adult animals grown on E. coli OP50 were transferred to the center of each bacterial lawn 429 after it. The number of animals on the bacterial lawns was counted at 12 and 24 hours after 430 exposure.

\section{Pharyngeal pumping rate assay}

433 Wild-type and unc-30(ok613) animals were synchronized by placing 20 gravid adult worms on 434 NGM plates seeded with E. coli OP50 and allowing them to lay eggs for $60 \mathrm{~min}$ at $20^{\circ} \mathrm{C}$. The 435 gravid adult worms were then removed, and the eggs were allowed to hatch and grow at $20^{\circ} \mathrm{C}$ 
until they reached the young adult stage. The synchronized worms were transferred to NGM plates fully seeded with P. aeruginosa for 24 hours at $25^{\circ} \mathrm{C}$. Worms were observed under the microscope with a focus on the pharynx. The number of contractions of the pharyngeal bulb was counted over $60 \mathrm{~s}$. Counting was conducted in triplicate and averaged to obtain pumping rates.

\section{Defecation rate assay}

442 Wild-type and mutant animals were synchronized by placing 20 gravid adult worms on NGM 443 plates seeded with $E$. coli OP50 and allowing them to lay eggs for $60 \mathrm{~min}$ at $20^{\circ} \mathrm{C}$. The gravid adult worms were then removed, and the eggs were allowed to hatch and grow at $20^{\circ} \mathrm{C}$ until they reached the young adult stage. The synchronized worms were transferred to NGM plates fully seeded with $P$. aeruginosa for 24 hours at $25^{\circ} \mathrm{C}$. Worms were observed under a microscope at room temperature. For each worm, an average of 10 intervals between two defecation cycles was measured. The defecation cycle was identified as a peristaltic contraction beginning at the posterior body of the animal and propagating to the anterior part of the animal followed by feces expulsion.

\section{Brood Size Assay}

453 The brood size assay was done following the earlier described methods (Kenyon et al., 1993;

454 Otarigho and Aballay, 2020). Ten L4 animals from egg-synchronized populations were 455 transferred to individual NGM plates (seeded with E. coli OP50) (described above) and 456 incubated at $20^{\circ} \mathrm{C}$. The animals were transferred to fresh plates every 24 hours. The progenies 457 were counted and removed every day.

\section{C. elegans Longevity Assays}

460 Longevity assays were performed on NGM plates containing live, heat killed, or UV-killed $E$.

461 coli strains HT115 or OP50 as described earlier (Otarigho and Aballay, 2020; Sun et al.,

462 2011)(Kumar et al., 2019; Sutphin and Kaeberlein, 2009). Animals were scored as alive, dead, or 463 gone each day. Animals that failed to display touch-provoked or pharyngeal movement were 464 scored as dead. Experimental groups contained 60 to 100 animals and the experiments were 465 performed in triplicate (Table 8). The assays were performed at $20^{\circ} \mathrm{C}$. 


\section{Intestinal Bacterial Loads Visualization and Quantification}

468 Intestinal bacterial loads were visualized and quantified as described earlier (Otarigho and Aballay, 2020; Sun et al., 2011). Briefly, P. aeruginosa-GFP lawns were prepared as described above. The plates were cooled to ambient temperature for at least an hour before seeding with young gravid adult hermaphroditic animals and the setup was placed at $25^{\circ} \mathrm{C}$ for 24 hours. The

472 animals were transferred from P. aeruginosa-GFP plates to the center of fresh E. coli plates for

$47310 \mathrm{~min}$ to eliminate $P$. aeruginosa-GFP on their body. The step was repeated two times more to

474 further eliminate external $P$. aeruginosa-GFP left from earlier steps. Subsequently, ten animals

475 were collected and used for fluorescence imaging to visualize the bacterial load while another ten 476 were transferred into $100 \mu \mathrm{L}$ of PBS plus $0.01 \%$ Triton X-100 and ground. Serial dilutions of the 477 lysates $\left(10^{1}-10^{10}\right)$ were seeded onto LB plates containing $50 \mu \mathrm{g} / \mathrm{mL}$ of kanamycin to select for $P$. aeruginosa-GFP cells and grown overnight at $37^{\circ} \mathrm{C}$. Single colonies were counted the next day and represented as the number of bacterial cells or CFU per animal.

\section{Fluorescence and bloating Imaging}

482 Fluorescence and bloating imaging was carried out as described previously (Otarigho and 483 Aballay, 2020; Singh and Aballay, 2019a, c). Briefly, animals were anesthetized using an M9 484 salt solution containing $50 \mathrm{mM}$ sodium azide and mounted onto $2 \%$ agar pads. The animals were then visualized for bacterial load using a Leica M165 FC fluorescence stereomicroscope. The diameter of the intestinal lumen was measured using Fiji-ImageJ software. At least 10 animals

\section{RNA Sequencing and Bioinformatic Analyses}

490 Approximately 40 gravid WT and unc-30(ok613) animals were placed for 3 hours on 10-cm

491 NGM plates (seeded with E. coli OP50) (described above) to have a synchronized population,

492 which developed and grew to L4 larval stage at $20^{\circ} \mathrm{C}$. Animals were washed off the plates with

493 M9 and frozen in QIAzol by ethanol/dry ice and stored at -80 prior to RNA extraction. Total

494 RNA was extracted using the RNeasy Plus Universal Kit (Qiagen, Netherlands). Residual

495 genomic DNA was removed using TURBO DNase (Life Technologies, Carlsbad, CA). A total of

$4966 \mu \mathrm{g}$ of total RNA was reverse-transcribed with random primers using the High-Capacity cDNA

497 Reverse Transcription Kit (Applied Biosystems, Foster City, CA). 
The library construction and RNA sequencing in Illumina NovaSeq 6000 platform was done following the method described by (Zhu et al., 2018) and (Yao et al., 2018) pair-end reads of $150 \mathrm{bp}$ were obtained for subsequent data analysis. The RNA sequence data were analyzed using a workflow constructed for Galaxy (https://usegalaxy.org) as described (Afgan et al., 2018; Afgan et al., 2016; Amrit and Ghazi, 2017). The RNA reads were aligned to the C. elegans genome (WS271) using the aligner STAR. Counts were normalized for sequencing depth and RNA composition across all samples. Differential gene expression analysis was then performed on normalized samples. Genes exhibiting at least two-fold change were considered differentially expressed. The differentially expressed genes were subjected SimpleMine tools from wormbase (https://www.wormbase.org/tools/mine/simplemine.cgi) to generate information such as wormBase ID and gene name, which are employed for further analyses. Gene ontology analysis was performed using the WormBase IDs in DAVID Bioinformatics Database (https://david.ncifcrf.gov) (Dennis et al., 2003) and validated using a C. elegans data enrichment analysis tool (https://wormbase.org/tools/enrichment/tea/tea.cgi). Immune pathways were obtained using the Worm Exp version 1 (http://wormexp.zoologie.uni-

513 kiel.de/wormexp/)(Yang et al., 2016). The Venn diagrams were obtained using the web tool

514 InteractiVenn (http://www.interactivenn.net) (Heberle et al., 2015) and bioinformatics and

515 evolutionary genomics tool (http://bioinformatics.psb.ugent.be/webtools/Venn/). Tissue-gene

516 expression prediction was used to simulate the expression pattern of UNC-30

517 (http://worm.princeton.edu) (Kaletsky et al., 2018). While neuron wiring was done using the

518 database of synaptic connectivity of C. elegans for computation (White et al., 1986)

519 http://ims.dse.ibaraki.ac.jp/ccep-tool/.

\section{RNA Isolation and Quantitative Reverse Transcription-PCR (qRT-PCR)}

522 Animals were synchronized and total RNA extraction was done following the protocol described 523 above. qRT-PCR was conducted using the Applied Biosystems One-Step Real-time PCR

524 protocol using SYBR Green fluorescence (Applied Biosystems) on an Applied Biosystems

525 7900HT real-time PCR machine in 96-well-plate format. Twenty-five-microliter reactions were

526 analyzed as outlined by the manufacturer (Applied Biosystems). The relative fold-changes of the 527 transcripts were calculated using the comparative $C T\left(2^{-\Delta \Delta C T}\right)$ method and normalized to pan-actin 528 (act-1, -3, -4). The cycle thresholds of the amplification were determined using StepOnePlus ${ }^{\mathrm{TM}}$ 
529 Real-Time PCR System Software v2.3 (Applied Biosystems). All samples were run in triplicate.

530 The primer sequences were available upon request and presented in Table S9.

532 Generation of transgenic C. elegans

533 To generate $u n c-30$ rescue animals, the $u n c-30$ DNA was amplified from the genomic DNA of

534 Bristol N2 C. elegans adult worms. Plasmid pPD160_Punc-30_unc-30 was constructed by

535 linearization of plasmid pPD160_Punc-30 using XbaI and SmaI restriction digestion enzymes.

536 The amplified unc-30 DNA was cloned behind its native promoter in the plasmid pPD160_Punc-

537 30, between XbaI and SmaI sites. For the neuronal rescue strain, the plasmid pPD95_77_Prab-

538 3_unc-30 was constructed by cloning the amplified unc-30 DNA into KpnI/HindIII digested

539 pPD95_77_Prab-3 under the promoter of $r a b-3$. The constructs were purified and sequenced.

540 Young adult hermaphrodite unc-30(ok613) C. elegans were transformed by microinjection of

541 plasmids into the gonad as described (Mello and Fire, 1995; Mello et al., 1991). Briefly, a

542 mixture containing pPD160_Punc-30_unc-30 (40 ng/ $\mu$ l) and Pmyo-3::mCherry pCFJ104 (5

$543 \mathrm{ng} / \mu \mathrm{l}$ ) that drives the expression of mCherry to the muscle as a transformation marker were

544 injected into the animals. For the neuronal rescue, a mixture containing pPD95_77_Prab-3_unc-

54530 plasmids $(40 \mathrm{ng} / \mu \mathrm{l})$ and Pmyo-2::mCherry $(5 \mathrm{ng} / \mu \mathrm{l})$ pCFJ90 that drives the expression of

546 mCherry to the pharynx as a transformation marker were injected into the animals.

547 The neuron ablated strains were generated by employing a two-component system

548 reconstituted caspase (recCaspase) for selective ablation of targeted cells (Chelur and Chalfie,

549 2007). The ASG ablated neuron was done by separately cloning the promoters of tax-2 and lim-6

550 in the recCaspases plasmids. Briefly, a 1817bp segment of the tax-2 promoter was cloned in the

551 CZ-ced-3(p17): )::unc-54 3'UTR plasmid at the Sph I and EcoR I site to generate the Ptax-

552 2::CZ::ced-3(p17)::unc-54 3'UTR plasmid, and a 5001bp segment of the Lim-6 promoter was

553 cloned in the ced-3(p15)+NZ:: unc-54 3'UTR plasmid at the Sph I and EcoR I site to generate

554 the Plim-6::ced-3(p15)-NZ::unc-54 3'UTR plasmid. A mixture containing Ptax-2-CZ-ced-

555 3(p17)-unc-54 3'UTR, Plim-6-ced-3(p15)-NZ-unc-54 3'UTR of 25ng/ $\mu \mathrm{L}$, and the roller marker

$556 \mathrm{pRF} 4$ rol-6(su1006) $(50 \mathrm{ng} / \mu \mathrm{L})$ was injected into the $\mathrm{N} 2$ young animals. To rescue unc-30 in

557 ASG neurons, gcy-15 promoter (1987bp) and unc-30 (5042bp) were amplified from gDNA. The

558 unc-30 fragment was cloned downstream of the Pgcy-15 by in-fusion PCR to obtain Pgcy-

$55915:$ unc-30, which was cloned into pPD95.77 between the Sph I and EcoR I sites to generate 
pPD95_77_Pgcy-15::unc-30. The ASG neuron-specific rescue strain unc-30(ok613);Pgcy15::unc-30 was generated by injecting plasmid pPD95_77_Pgcy-15::unc-30 (25 ng/ $\mu \mathrm{l})$ with Pmyo-2::mCherry $(10 \mathrm{ng} / \mu \mathrm{l})$ as a co-injection marker into the unc-30(ok613) animals.

The PVP ablation was done by separately cloning the promoters of $o d r-2 \mathrm{~b}$ isoform and unc-53 in the recCaspases plasmids. Briefly, a 2661bp segment of the $o d r-2 \mathrm{~b}$ isoform was cloned in the CZ::ced-3(p17)::unc-54 3'UTR plasmid at the Sph I and EcoR I site to obtain the Podr-2b::CZ::ced-3(p17)::unc-54 3'UTR plasmid and a segment of 3012bp of unc-53 was 567 cloned in the ced-3(p15)-NZ::unc-54 3'UTR plasmid at the Sph I and EcoR I site to obtain the Punc-53-ced-3(p15)-NZ-unc-54 3'UTR plasmid. A mixture containing $25 \mathrm{ng} / \mu \mathrm{L}$ of the Podr- $2 b$ CZ-ced-3(p17)-unc-54 3'UTR plasmid, 25ng/ $\mu \mathrm{L}$ of the Punc-53-ced-3(p15)-NZ-unc-54 3'UTR plasmid, and 50ng/ $\mu \mathrm{L}$ of the roller marker pRF4 rol-6(su1006) was injected into $\mathrm{N} 2$ young animals. The successful transformation was determined by the identification of the selection marker. At least three independent lines carrying extrachromosomal arrays were obtained for each construct. Only worms with dominant roller phenotypes were selected for further experiment. Primers used in the generation of transgenic animals are shown in Table S9.

\section{Quantification and Statistical Analysis}

Statistical analysis was performed with Prism 8 version 8.1.2 (GraphPad). All error bars represent the standard deviation (SD). The two-sample t test was used when needed, and the data were judged to be statistically significant when $\mathrm{p}<0.05$. In the figures, asterisks $(*)$ denote statistical significance as follows: ns, not significant, ${ }^{*}, \mathrm{p}<0.05, * *, \mathrm{p}<0.001, * * *, \mathrm{p}<0.0001$, as compared with the appropriate controls. The Kaplan-Meier method was used to calculate the survival fractions, and statistical significance between survival curves was determined using the

\section{ACKNOWLEDGMENTS}

586 This work was fully supported by NIH grants GM0709077 and AI117911 (to A.A.). Most strains

587 used in this study were obtained from the Caenorhabditis Genetics Center (CGC), which is

588 funded by the NIH Office of Research Infrastructure Programs (P40 OD010440) and the

589 National BioResource Project (NBRP) of Japan. 


\section{AUTHOR CONTRIBUTIONS}

592 B.O and A.A. conceived and designed the experiments. B.O. performed the experiments. B.O. and A.A. analyzed the data and wrote the paper.

\section{DECLARATION OF INTERESTS}

The authors declare no competing interests.

\section{References}

Aballay, A., Drenkard, E., Hilbun, L.R., and Ausubel, F.M. (2003). Caenorhabditis elegans innate immune response triggered by Salmonella enterica requires intact LPS and is mediated by a MAPK signaling pathway. Current Biology 13, 47-52. Afgan, E., Baker, D., Batut, B., Van Den Beek, M., Bouvier, D., Čech, M., Chilton, J., Clements, D., Coraor, N., and Grüning, B.A. (2018). The Galaxy platform for accessible, reproducible and collaborative biomedical analyses: 2018 update. Nucleic acids research 46, W537-W544. Afgan, E., Baker, D., Van den Beek, M., Blankenberg, D., Bouvier, D., Čech, M., Chilton, J., Clements, D., Coraor, N., and Eberhard, C. (2016). The Galaxy platform for accessible, reproducible and collaborative biomedical analyses: 2016 update. Nucleic acids research 44, W3-W10.

Alcedo, J., and Kenyon, C. (2004). Regulation of C. elegans longevity by specific gustatory and olfactory neurons. Neuron 41, 45-55.

Amrit, F.R., and Ghazi, A. (2017). Transcriptomic Analysis of C. elegans RNA Sequencing Data Through the Tuxedo Suite on the Galaxy Project. JoVE (Journal of Visualized Experiments), e55473.

Amrit, F.R., Naim, N., Ratnappan, R., Loose, J., Mason, C., Steenberge, L., McClendon, B.T., Wang, G., Driscoll, M., and Yanowitz, J.L. (2019). The longevity-promoting factor, TCER-1, widely represses stress resistance and innate immunity. Nature communications 10, 1-16.

Anderson, A., Laurenson-Schafer, H., Partridge, F.A., Hodgkin, J., and McMullan, R. (2013). Serotonergic chemosensory neurons modify the C. elegans immune response by regulating Gprotein signaling in epithelial cells. PLoS Pathog 9, e1003787.

Angeles-Albores, D., Lee, R.Y., Chan, J., and Sternberg, P.W. (2016). Tissue enrichment analysis for C. elegans genomics. BMC bioinformatics 17, 366.

Apfeld, J., and Kenyon, C. (1999). Regulation of lifespan by sensory perception in Caenorhabditis elegans. Nature 402, 804-809.

Ayres, J.S. (2020). Immunometabolism of infections. Nature Reviews Immunology 20, 79-80. Backhed, F., Bugianesi, E., Christofk, H., Dikic, I., Gupta, R., Mair, W.B., O’Neill, L.A., Ralser, M., Sabatini, D.M., and Tschop, M. (2019). The next decade of metabolism. Nat Metabolism 1, 2-4.

Bargmann, C.I. (2006). Chemosensation in C. elegans. In WormBook: The Online Review of C. elegans Biology [Internet] (WormBook).

631 regulation of DAF-16 by kri-1 and lipophilic-hormone signaling. Cell 124, 1055-1068. Bird, L. (2019). Getting enough energy for immunity. Nature Reviews Immunology 19, 269-269. 
Bolz, D.D., Tenor, J.L., and Aballay, A. (2010). A conserved PMK-1/p38 MAPK is required in Caenorhabditis elegans tissue-specific immune response to Yersinia pestis infection. Journal of Biological Chemistry 285, 10832-10840. Brenner, S. (1974). The genetics of Caenorhabditis elegans. Genetics 77, 71-94. Cao, X., and Aballay, A. (2016). Neural inhibition of dopaminergic signaling enhances immunity in a cell-non-autonomous manner. Current Biology 26, 2329-2334. Cao, X., Kajino-Sakamoto, R., Doss, A., and Aballay, A. (2017). Distinct roles of sensory neurons in mediating pathogen avoidance and neuropeptide-dependent immune regulation. Cell stress enzymes are required for DAF-16-mediated immunity due to generation of reactive oxygen species by Caenorhabditis elegans. Genetics 176, 1567-1577.

Chelur, D.S., and Chalfie, M. (2007). Targeted cell killing by reconstituted caspases.

Proceedings of the National Academy of Sciences 104, 2283-2288.

Chun, L., Gong, J., Yuan, F., Zhang, B., Liu, H., Zheng, T., Yu, T., Xu, X.S., and Liu, J. (2015). Metabotropic GABA signalling modulates longevity in C. elegans. Nature communications 6, 110.

Cinar, H., Keles, S., and Jin, Y. (2005). Expression profiling of GABAergic motor neurons in Caenorhabditis elegans. Current Biology 15, 340-346.

Dennis, G., Sherman, B.T., Hosack, D.A., Yang, J., Gao, W., Lane, H.C., and Lempicki, R.A. (2003). DAVID: database for annotation, visualization, and integrated discovery. Genome biology 4, R60.

Eastman, C., Horvitz, H.R., and Jin, Y. (1999a). Coordinated Transcriptional Regulation of the unc-25Glutamic Acid Decarboxylase and the unc-47 GABA Vesicular Transporter by the Caenorhabditis elegans UNC-30 Homeodomain Protein. Journal of Neuroscience 19, 6225-6234. Eastman, C., Horvitz, H.R., and Jin, Y. (1999b). Coordinated Transcriptional Regulation of the unc-25Glutamic Acid Decarboxylase and the unc-47 GABA Vesicular Transporter by the Caenorhabditis elegans UNC-30 Homeodomain Protein. Journal of Neuroscience 19, 6225-6234. Franceschi, C., Garagnani, P., Parini, P., Giuliani, C., and Santoro, A. (2018). Inflammaging: a new immune-metabolic viewpoint for age-related diseases. Nature Reviews Endocrinology 14, 576-590.

Ganeshan, K., and Chawla, A. (2014). Metabolic regulation of immune responses. Annual review of immunology 32, 609-634.

Ganeshan, K., Nikkanen, J., Man, K., Leong, Y.A., Sogawa, Y., Maschek, J.A., Van Ry, T., Chagwedera, D.N., Cox, J.E., and Chawla, A. (2019). Energetic trade-offs and hypometabolic states promote disease tolerance. Cell 177, 399-413. e312.

Garcia, S.M., Casanueva, M.O., Silva, M.C., Amaral, M.D., and Morimoto, R.I. (2007). Neuronal signaling modulates protein homeostasis in Caenorhabditis elegans post-synaptic muscle cells. Genes \& development 21, 3006-3016.

Garigan, D., Hsu, A.-L., Fraser, A.G., Kamath, R.S., Ahringer, J., and Kenyon, C. (2002). Genetic analysis of tissue aging in Caenorhabditis elegans: a role for heat-shock factor and bacterial proliferation. Genetics $161,1101-1112$.

Garsin, D.A., Villanueva, J.M., Begun, J., Kim, D.H., Sifri, C.D., Calderwood, S.B., Ruvkun, G., and Ausubel, F.M. (2003). Long-lived C. elegans daf-2 mutants are resistant to bacterial pathogens. Science 300, 1921-1921. 
677 Gendrel, M., Atlas, E.G., and Hobert, O. (2016). A cellular and regulatory map of the

678 GABAergic nervous system of C. elegans. Elife 5, e17686.

679 Hajdu-Cronin, Y.M., Chen, W.J., and Sternberg, P.W. (2004). The L-type cyclin CYL-1 and the heat-shock-factor HSF-1 are required for heat-shock-induced protein expression in Caenorhabditis elegans. Genetics 168, 1937-1949. Head, B.P., Olaitan, A.O., and Aballay, A. (2017). Role of GATA transcription factor ELT-2 and p38 MAPK PMK-1 in recovery from acute P. aeruginosa infection in C. elegans. Virulence 8 , 261-274.

Heberle, H., Meirelles, G.V., da Silva, F.R., Telles, G.P., and Minghim, R. (2015). InteractiVenn: a web-based tool for the analysis of sets through Venn diagrams. BMC bioinformatics 16, 169 . Hoffman, C., and Aballay, A. (2019). Role of neurons in the control of immune defense. Current opinion in immunology 60, 30-36. Huffman, D.L., Abrami, L., Sasik, R., Corbeil, J., van der Goot, F.G., and Aroian, R.V. (2004). Mitogen-activated protein kinase pathways defend against bacterial pore-forming toxins. Proceedings of the National Academy of Sciences 101, 10995-11000. Jeong, D.-E., Artan, M., Seo, K., and Lee, S.-J. (2012). Regulation of lifespan by chemosensory and thermosensory systems: findings in invertebrates and their implications in mammalian aging. Frontiers in genetics 3, 218.

Jin, Y., Hoskins, R., and Horvitz, H.R. (1994). Control of type-D GABAergic neuron differentiation by C. elegans UNC-30 homeodomain protein. Nature 372, 780-783.

Johnson, D.W., Llop, J.R., Farrell, S.F., Yuan, J., Stolzenburg, L.R., and Samuelson, A.V. (2014). The Caenorhabditis elegans Myc-Mondo/Mad complexes integrate diverse longevity signals. PLoS Genet 10, e1004278. Jung, J., Zeng, H., and Horng, T. (2019). Metabolism as a guiding force for immunity. Nature cell biology 21, 85-93.

704 Kaiser, A., Schmidt, M., Huber, O., Frietsch, J.J., Scholl, S., Heidel, F.H., Hochhaus, A., Müller, J.P., and Ernst, T. (2020). SIRT7: an influence factor in healthy aging and the development of age-dependent myeloid stem-cell disorders. Leukemia, 1-11.

Kaletsky, R., Yao, V., Williams, A., Runnels, A.M., Tadych, A., Zhou, S., Troyanskaya, O.G., and Murphy, C.T. (2018). Transcriptome analysis of adult Caenorhabditis elegans cells reveals tissue-specific gene and isoform expression. PLoS genetics 14, e1007559.

Kenyon, C., Chang, J., Gensch, E., Rudner, A., and Tabtiang, R. (1993). A C. elegans mutant that lives twice as long as wild type. Nature 366, 461.

Kenyon, C.J. (2010). The genetics of ageing. Nature 464, 504-512.

Kerry, S., TeKippe, M., Gaddis, N.C., and Aballay, A. (2006). GATA transcription factor required for immunity to bacterial and fungal pathogens. PLoS One 1, e77.

Kim, D.H., Feinbaum, R., Alloing, G., Emerson, F.E., Garsin, D.A., Inoue, H., Tanaka-Hino, M., Hisamoto, N., Matsumoto, K., and Tan, M.-W. (2002). A conserved p38 MAP kinase pathway in Caenorhabditis elegans innate immunity. Science 297, 623-626.

Kuchroo, V.K., Ohashi, P.S., Sartor, R.B., and Vinuesa, C.G. (2012). Dysregulation of immune homeostasis in autoimmune diseases. Nature medicine 18, 42-47.

Kumar, S., Egan, B.M., Kocsisova, Z., Schneider, D.L., Murphy, J.T., Diwan, A., and Kornfeld, K. (2019). Lifespan extension in C. elegans caused by bacterial colonization of the intestine and subsequent activation of an innate immune response. Developmental cell 49, 100-117. e106. 
Kurup, N., and Jin, Y. (2016). Neural circuit rewiring: insights from DD synapse remodeling. In Worm (Taylor \& Francis), p. e1129486. Labed, S.A., Wani, K.A., Jagadeesan, S., Hakkim, A., Najibi, M., and Irazoqui, J.E. (2018). Intestinal epithelial Wnt signaling mediates acetylcholine-triggered host defense against infection. Immunity 48, 963-978. e963.

Li, C., and Kim, K. (2008). Neuropeptides. WormBook: the online review of C. elegans biology, 1. pathogen resistance in Drosophila melanogaster are mediated by NFkB signaling. Aging cell 5, 533-543. in the regulation of lifespan. Cell 115, 489-502.

McIntire, S.L., Reimer, R.J., Schuske, K., Edwards, R.H., and Jorgensen, E.M. (1997). Identification and characterization of the vesicular GABA transporter. Nature 389, 870-876. Mclntire, S.L., Jorgensen, E., and Horvitz, H.R. (1993a). Genes required for GABA function in Caenorhabditis elegans. Nature 364, 334-337.

738 Mclntire, S.L., Jorgensen, E., Kaplan, J., and Horvitz, H.R. (1993b). The GABAergic nervous system of Caenorhabditis elegans. Nature 364, 337-341.

Mello, C., and Fire, A. (1995). DNA transformation. In Methods in cell biology (Elsevier), pp. 451-482.

Mello, C.C., Kramer, J.M., Stinchcomb, D., and Ambros, V. (1991). Efficient gene transfer in C. elegans: extrachromosomal maintenance and integration of transforming sequences. The EMBO journal 10,3959-3970.

Mohri-Shiomi, A., and Garsin, D.A. (2008). Insulin signaling and the heat shock response modulate protein homeostasis in the Caenorhabditis elegans intestine during infection. Journal of Biological Chemistry 283, 194-201.

Morris, J.Z., Tissenbaum, H.A., and Ruvkun, G. (1996). A phosphatidylinositol-3-OH kinase family member regulating longevity and diapause in Caenorhabditis elegans. Nature 382, 536539.

Murphy, C.T., McCarroll, S.A., Bargmann, C.I., Fraser, A., Kamath, R.S., Ahringer, J., Li, H., and Kenyon, C. (2003). Genes that act downstream of DAF-16 to influence the lifespan of Caenorhabditis elegans. Nature 424, 277-283.

Nakad, R., Snoek, L.B., Yang, W., Ellendt, S., Schneider, F., Mohr, T.G., Rösingh, L., Masche, A.C., Rosenstiel, P.C., and Dierking, K. (2016). Contrasting invertebrate immune defense behaviors caused by a single gene, the Caenorhabditis elegans neuropeptide receptor gene npr-1. BMC genomics 17, 1-20.

Nakamura, S., Karalay, Ö., Jäger, P.S., Horikawa, M., Klein, C., Nakamura, K., Latza, C., Templer, S.E., Dieterich, C., and Antebi, A. (2016). Mondo complexes regulate TFEB via TOR inhibition to promote longevity in response to gonadal signals. Nature communications 7, 1-15. Otarigho, B., and Aballay, A. (2020). Cholesterol regulates innate immunity via nuclear hormone receptor NHR-8. iScience.

Pereira, L., Kratsios, P., Serrano-Saiz, E., Sheftel, H., Mayo, A.E., Hall, D.H., White, J.G., LeBoeuf, B., Garcia, L.R., and Alon, U. (2015). A cellular and regulatory map of the cholinergic nervous system of C. elegans. Elife 4, e12432.

Pinoli, M., Marino, F., and Cosentino, M. (2017). Dopaminergic regulation of innate immunity: a review. Journal of Neuroimmune Pharmacology 12, 602-623. 
Pocock, R., and Hobert, O. (2010). Hypoxia activates a latent circuit for processing gustatory information in C. elegans. Nature neuroscience 13, 610. Powell, J.R., and Ausubel, F.M. (2008). Models of Caenorhabditis elegans infection by bacterial and fungal pathogens. In Innate immunity (Springer), pp. 403-427. Rajan, M., Anderson, C.P., Rindler, P.M., Romney, S.J., dos Santos, M.C.F., Gertz, J., and Leibold, E.A. (2019). NHR-14 loss of function couples intestinal iron uptake with innate immunity in C. elegans through PQM-1 signaling. In eLife, p. e44674. Reddy, K.C., Andersen, E.C., Kruglyak, L., and Kim, D.H. (2009). A polymorphism in npr-1 is a behavioral determinant of pathogen susceptibility in C. elegans. Science 323, 382-384. Reddy, K.C., Hunter, R.C., Bhatla, N., Newman, D.K., and Kim, D.H. (2011). Caenorhabditis elegans NPR-1-mediated behaviors are suppressed in the presence of mucoid bacteria. Proceedings of the National Academy of Sciences 108, 12887-12892.

Rex, E., Hapiak, V., Hobson, R., Smith, K., Xiao, H., and Komuniecki, R. (2005). TYRA-2 (F01E11. 5): a Caenorhabditis elegans tyramine receptor expressed in the MC and NSM pharyngeal neurons. Journal of neurochemistry 94, 181-191.

Riera, C.E., and Dillin, A. (2015). Tipping the metabolic scales towards increased longevity in mammals. Nature cell biology 17, 196-203.

Riesen, M., Feyst, I., Rattanavirotkul, N., Ezcurra, M., Tullet, J.M., Papatheodorou, I., Ziehm, M., Au, C., Gilliat, A.F., and Hellberg, J. (2014). MDL-1, a growth-and tumor-suppressor, slows aging and prevents germline hyperplasia and hypertrophy in C. elegans. Aging (Albany NY) 6, 98.

Sellegounder, D., Liu, Y., Wibisono, P., Chen, C.-H., Leap, D., and Sun, J. (2019). Neuronal GPCR NPR-8 regulates C. elegans defense against pathogen infection. Science Advances 5, eaaw4717.

Shapira, M., Hamlin, B.J., Rong, J., Chen, K., Ronen, M., and Tan, M.-W. (2006). A conserved role for a GATA transcription factor in regulating epithelial innate immune responses.

Proceedings of the National Academy of Sciences 103, 14086-14091.

Sifri, C.D., Begun, J., Ausubel, F.M., and Calderwood, S.B. (2003). Caenorhabditis elegans as a model host for Staphylococcus aureus pathogenesis. Infection and immunity 71, 2208-2217. Singh, J., and Aballay, A. (2019a). Intestinal infection regulates behavior and learning via neuroendocrine signaling. eLife 8 .

Singh, J., and Aballay, A. (2019b). Microbial colonization activates an immune fight-and-flight response via neuroendocrine signaling. Developmental cell 49, 89-99. e84.

Singh, J., and Aballay, A. (2019c). Microbial colonization activates an immune fight-and-flight response via neuroendocrine signaling. Developmental cell 49, 89-99. e84.

Singh, V., and Aballay, A. (2006). Heat-shock transcription factor (HSF)-1 pathway required for Caenorhabditis elegans immunity. Proceedings of the National Academy of Sciences 103, 13092-13097.

Singh, V., and Aballay, A. (2009). Regulation of DAF-16-mediated Innate Immunity in Caenorhabditis elegans. Journal of Biological Chemistry 284, 35580-35587.

Solana, R., Pawelec, G., and Tarazona, R. (2006). Aging and innate immunity. Immunity 24, 491-494.

Styer, K.L., Singh, V., Macosko, E., Steele, S.E., Bargmann, C.I., and Aballay, A. (2008). Innate immunity in Caenorhabditis elegans is regulated by neurons expressing NPR-1/GPCR. Science $322,460-464$. 
Sugiura, M., Fuke, S., Suo, S., Sasagawa, N., Van Tol, H.H., and Ishiura, S. (2005).

Characterization of a novel D2-like dopamine receptor with a truncated splice variant and a D1like dopamine receptor unique to invertebrates from Caenorhabditis elegans. Journal of neurochemistry 94, 1146-1157. immunity by regulating noncanonical unfolded protein response genes. Science 332, 729-732. Sutphin, G.L., and Kaeberlein, M. (2009). Measuring Caenorhabditis elegans life span on solid media. JoVE (Journal of Visualized Experiments), e1152.

Tan, M.-W., Mahajan-Miklos, S., and Ausubel, F.M. (1999). Killing of Caenorhabditis elegans by Pseudomonas aeruginosa used to model mammalian bacterial pathogenesis. Proceedings of the National Academy of Sciences 96, 715-720.

Templeman, N.M., Luo, S., Kaletsky, R., Shi, C., Ashraf, J., Keyes, W., and Murphy, C.T. (2018). Insulin signaling regulates oocyte quality maintenance with age via cathepsin B activity. Current Biology 28, 753-760. e754.

Tepper, R.G., Ashraf, J., Kaletsky, R., Kleemann, G., Murphy, C.T., and Bussemaker, H.J. (2013a). PQM-1 complements DAF-16 as a key transcriptional regulator of DAF-2-mediated development and longevity. In Cell, pp. 676-690.

830 Tepper, R.G., Ashraf, J., Kaletsky, R., Kleemann, G., Murphy, C.T., and Bussemaker, H.J. 831 (2013b). PQM-1 complements DAF-16 as a key transcriptional regulator of DAF-2-mediated development and longevity. Cell 154, 676-690.

Tepper, R.G., Murphy, C.T., and Bussemaker, H.J. (2014). DAF-16 and PQM-1: partners in longevity. Aging (Albany NY) 6, 5.

Tracey, K.J. (2002). The inflammatory reflex. Nature 420, 853-859.

Troemel, E.R., Chu, S.W., Reinke, V., Lee, S.S., Ausubel, F.M., and Kim, D.H. (2006). p38 MAPK regulates expression of immune response genes and contributes to longevity in C. elegans. PLoS genetics 2, e183. Tullet, J.M., Hertweck, M., An, J.H., Baker, J., Hwang, J.Y., Liu, S., Oliveira, R.P., Baumeister, R., and Blackwell, T.K. (2008). Direct inhibition of the longevity-promoting factor SKN-1 by insulin-like signaling in C. elegans. Cell 132, 1025-1038.

Walton, T., Preston, E., Nair, G., Zacharias, A.L., Raj, A., and Murray, J.I. (2015). The Bicoid class homeodomain factors ceh-36/OTX and unc-30/PITX cooperate in C. elegans embryonic progenitor cells to regulate robust development. PLoS genetics 11 .

Wani, K.A., Goswamy, D., and Irazoqui, J.E. (2019). A Nutrition-Longevity Tradeoff Enforced by Innate Immunity. Molecular cell 74, 864-865.

Westmoreland, J.J., McEwen, J., Moore, B.A., Jin, Y., and Condie, B.G. (2001). Conserved Function of Caenorhabditis elegansUNC-30 and Mouse Pitx2 in Controlling GABAergic Neuron Differentiation. Journal of Neuroscience 21, 6810-6819.

White, J.G., Southgate, E., Thomson, J.N., and Brenner, S. (1986). The structure of the nervous system of the nematode Caenorhabditis elegans. Philos Trans R Soc Lond B Biol Sci 314, 1-340. Wolkow, C.A., Kimura, K.D., Lee, M.-S., and Ruvkun, G. (2000). Regulation of C. elegans lifespan by insulinlike signaling in the nervous system. Science 290, 147-150.

Wray, C., and Sojka, W. (1978). Experimental Salmonella typhimurium infection in calves. Research in veterinary science $25,139-143$.

Wu, Z., Isik, M., Moroz, N., Steinbaugh, M.J., Zhang, P., and Blackwell, T.K. (2019). Dietary restriction extends lifespan through metabolic regulation of innate immunity. Cell metabolism

858 29, 1192-1205. e1198. 
859 Yang, W., Dierking, K., and Schulenburg, H. (2016). WormExp: a web-based application for a 860 Caenorhabditis elegans-specific gene expression enrichment analysis. Bioinformatics 32, 943-

861945.

862 Yao, L., Wang, J., Li, B., Meng, Y., Ma, X., Si, E., Ren, P., Yang, K., Shang, X., and Wang, H.

863 (2018). Transcriptome sequencing and comparative analysis of differentially-expressed isoforms

864 in the roots of Halogeton glomeratus under salt stress. Gene 646, 159-168.

865 Yuan, F., Zhou, J., Xu, L., Jia, W., Chun, L., Xu, X.S., and Liu, J. (2019). GABA receptors

866 differentially regulate life span and health span in C. elegans through distinct downstream

867 mechanisms. American Journal of Physiology-Cell Physiology 317, C953-C963.

868 Zhu, F.-Y., Chen, M.-X., Ye, N.-H., Qiao, W.-M., Gao, B., Law, W.-K., Tian, Y., Zhang, D.,

869 Zhang, D., and Liu, T.-Y. (2018). Comparative performance of the BGISEQ-500 and Illumina

870 HiSeq4000 sequencing platforms for transcriptome analysis in plants. Plant methods 14, 69.

871 
bioRxiv preprint doi: https://doi.org/10.1101/2021.02.25.432801; this version posted February 26, 2021. The copyright holder for this preprint (which was not certified by peer review) is the author/funder. All rights reserved. No reuse allowed without permission.

A
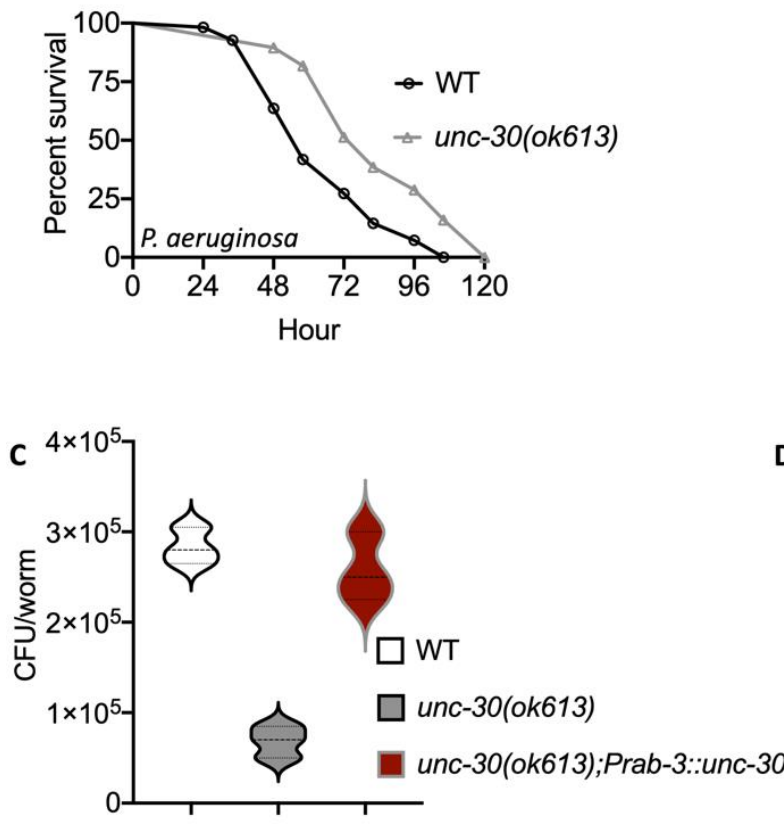

E

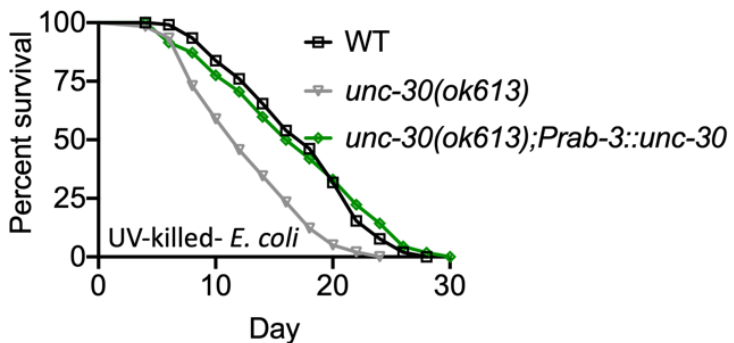

G

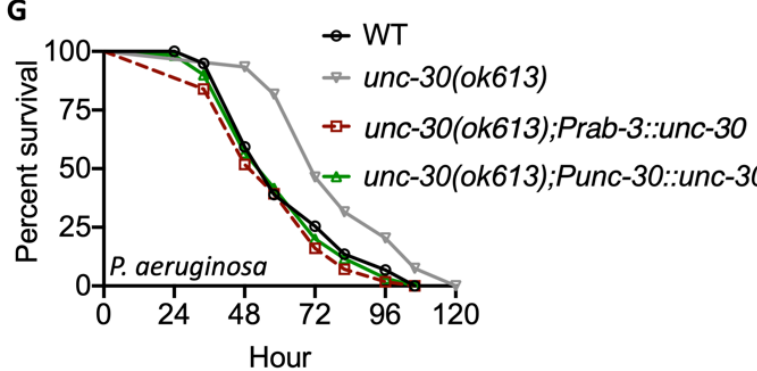

I

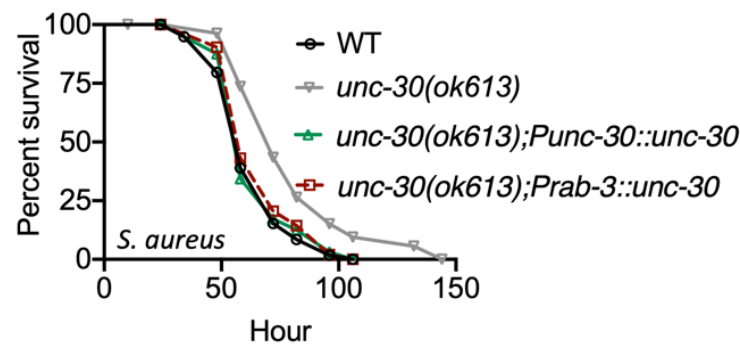

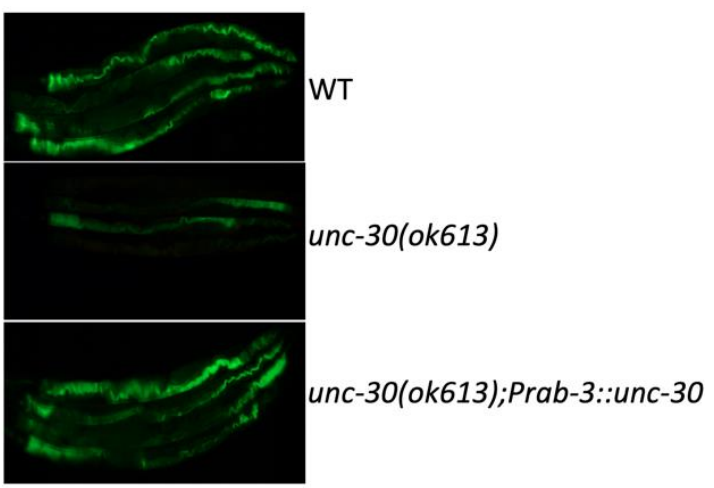

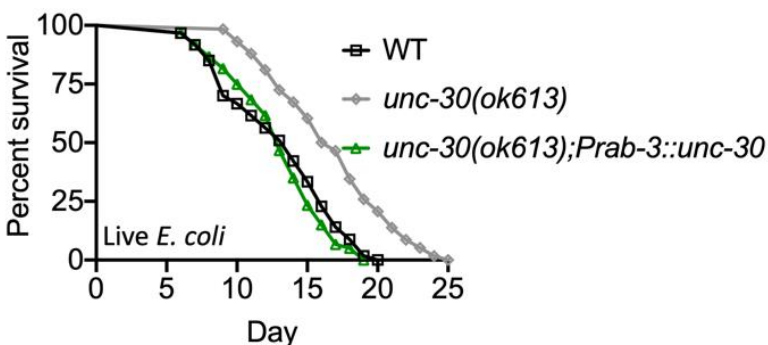

$\mathbf{F}$
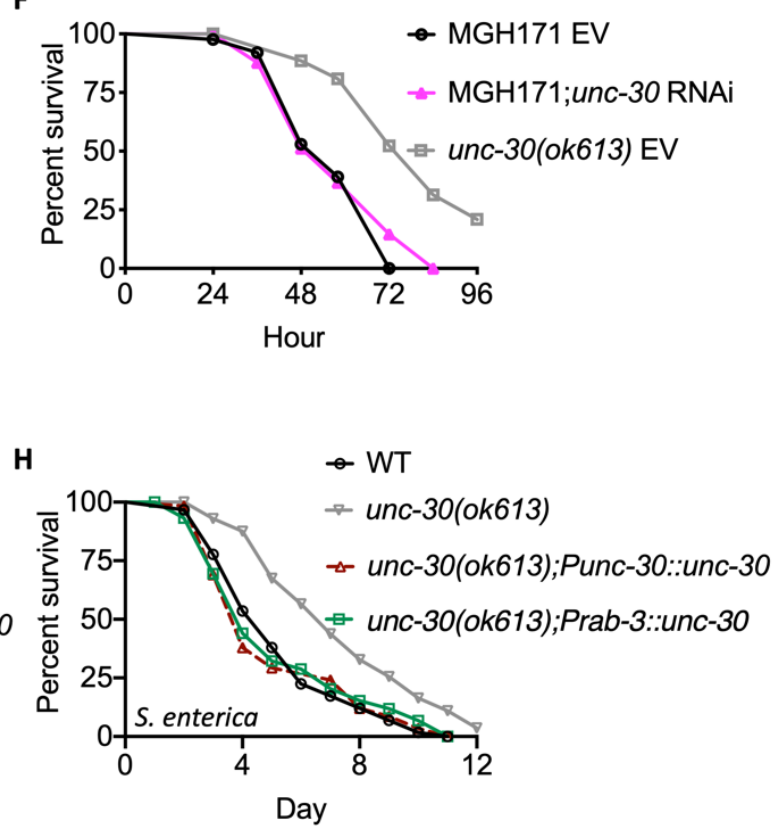

J

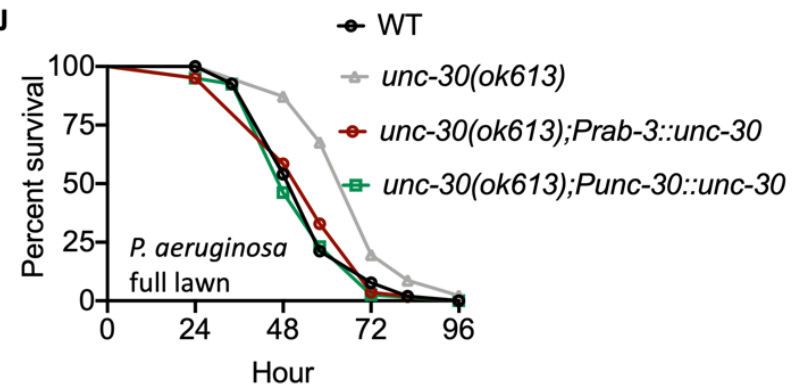

Figure 1. UNC-30 Functional Loss exhibits enhanced immunity and reduced longevity.

(A) Wild type (WT) and unc-30(ok613) animals were exposed to P. aeruginosa partial lawn and scored for survival. WT vs unc-30(ok613), P $<0.0001$.

(B) Colonization of WT, unc-30(ok613), and unc-30(ok613);Prab-3::unc-30 animals by P. aeruginosa-GFP after 24 hours at $25^{\circ} \mathrm{C}$. 
bioRxiv preprint doi: https://doi.org/10.1101/2021.02.25.432801; this version posted February 26, 2021. The copyright holder for this preprint (which was not certified by peer review) is the author/funder. All rights reserved. No reuse allowed without permission.

(C) Colony-forming units per animal [WT, unc-30(ok613), and unc-30(ok613);Prab$3:: u n c-30]$ grown on P. aeruginosa-GFP for 24 hours at $25^{\circ} \mathrm{C}$. Bars represent means while error bars indicate SD; $* * * * \mathrm{p}<0.001$.

(D) WT, unc-30(ok613), and unc-30(ok613);Prab-3::unc-30 animals were exposed live E. coli and scored for survival. WT vs unc-30(ok613), P < 0.0001; unc-30(ok613);Prab$3::$ unc-30, $\mathrm{P}=\mathrm{NS}$.

(E) WT, unc-30(ok613), and unc-30(ok613);Prab-3::unc-30 animals were exposed UVkilled $E$. coli and scored for survival. WT vs unc-30(ok613), P < 0.0001; unc30(ok613);Prab-3::unc-30, P=NS.

(F) RNAi intestine-specific strain MGH171, MGH171; unc-30 RNAi, and unc-30(ok613) animals were exposed to $P$. aeruginosa and scored for survival. EV, empty vector RNAi control. MGH171 EV vs. unc-30(ok613) EV, P < 0.0001; MGH171;unc-30 RNAi, $\mathrm{P}=\mathrm{NS}$.

(G) WT, unc-30(ok613), unc-30(ok613);Punc-30::unc-30, and unc-30(ok613);Prab$3::$ unc-30 animals were exposed to $P$. aeruginosa partial lawn and scored for survival. WT vs unc-30(ok613), $\mathrm{P}<0.0001$; unc-30(ok613);Punc-30::unc-30, and unc-30(ok613);Prab-3::unc-30, P = NS.

(H) WT, unc-30(ok613), unc-30(ok613);Punc-30::unc-30, and unc-30(ok613);Prab$3::$ unc-30 animals were exposed $S$. enterica partial lawn and scored for survival. WT vs unc-30(ok613), $\mathrm{P}<0.0001$; unc-30(ok613);Punc-30::unc-30, and unc30(ok613);Prab-3::unc-30, P = NS.

(I) WT, unc-30(ok613), unc-30(ok613);Punc-30::unc-30, and unc-30(ok613);Prab$3:: u n c-30$ animals were exposed $S$. aureus partial lawn and scored for survival. WT vs unc-30(ok613), $\mathrm{P}<0.0001$; unc-30(ok613);Punc-30::unc-30, and unc30(ok613);Prab-3::unc-30, P = NS.

(J) WT, unc-30(ok613), unc-30(ok613);Punc-30::unc-30, and unc-30(ok613);Prab$3::$ unc-30 animals were exposed to $P$. aeruginosa full lawn and scored for survival. WT vs unc-30(ok613), $\mathrm{P}<0.0001$; unc-30(ok613);Punc-30::unc-30, and unc30(ok613);Prab-3::unc-30, P = NS. 


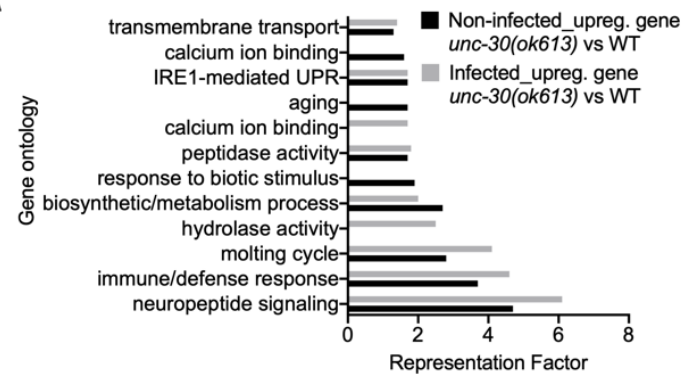

C
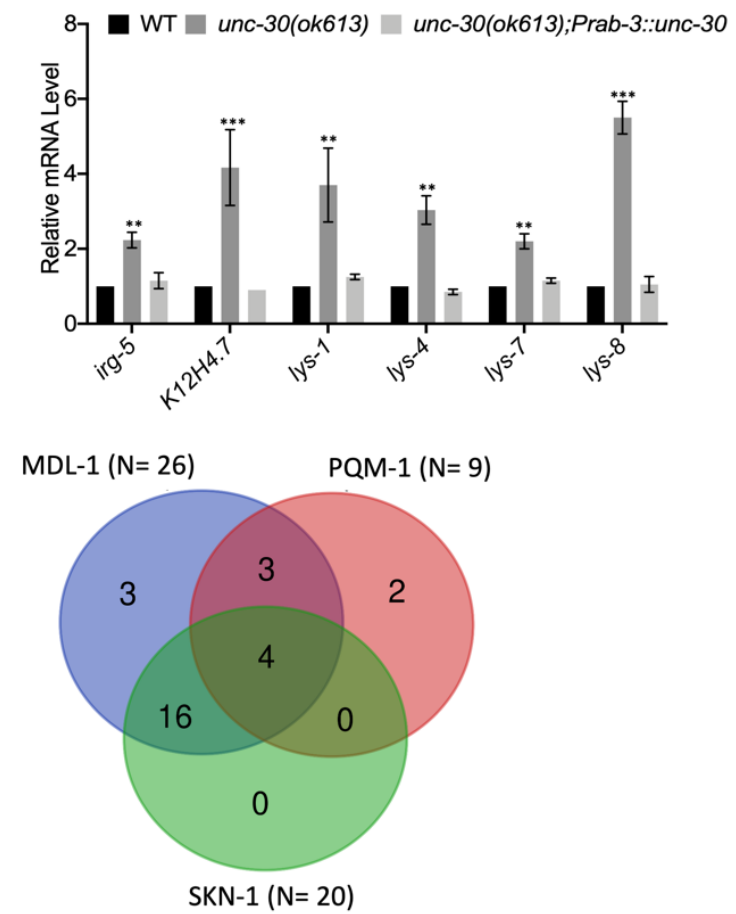

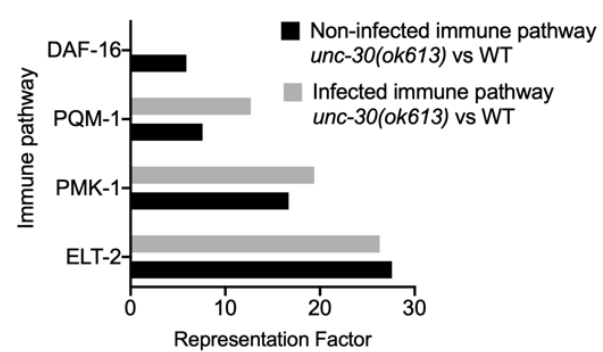

D

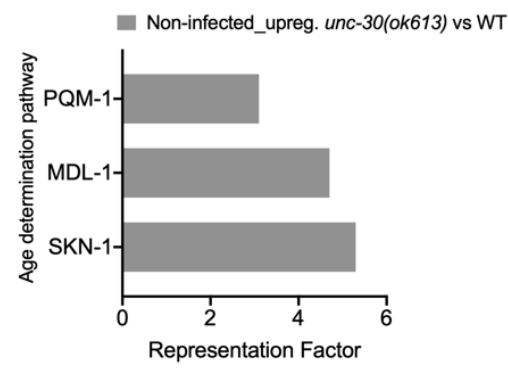

$\mathbf{F}$

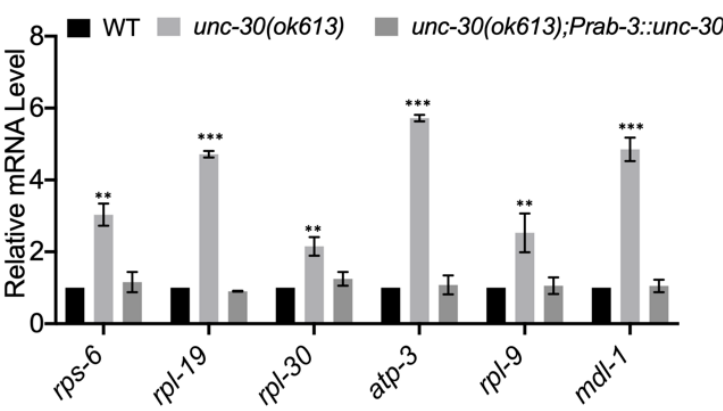

Figure 2. PITX1/UNC-30 regulates immune and age determination pathways

(A) Gene ontology analysis of upregulated genes in unc-30(ok613) vs WT in both noninfected and $P$. aeruginosa-infected animals. The cutoff is based on the filtering thresholds of $\mathrm{P}<0.05$ and arranged according to the representation factor.

(B) Representation factors of immune pathways for the upregulated immune genes in unc30 (ok613) vs WT in both non-infected and $P$. aeruginosa-infected animals.

(C) qRT-PCR analysis of immune gene expression in WT and unc-30(ok613) animals. Bars represent means while error bars indicate SD; *p $<0.05$, ** $<0.001$ and ***p $<$ 0.0001 .

(D) Representation factors of age determination pathways for the upregulated aging genes in unc-30(ok613) vs WT in non-infected animals.

(E) Venn diagram showing the age determination genes in each pathway for the upregulated aging genes in unc-30(ok613) vs WT in non-infected animals.

(F) qRT-PCR analysis of age determination genes expression in WT and unc-30(ok613) animals. Bars represent means while error bars indicate SD; $* p<0.05, * * p<0.001$ and $* * * p<0.0001$. 
A
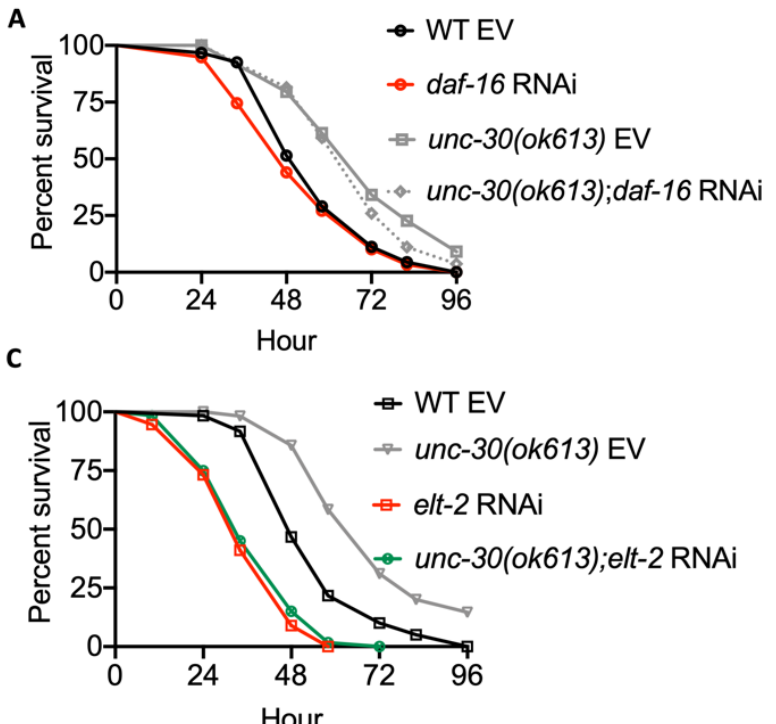

E

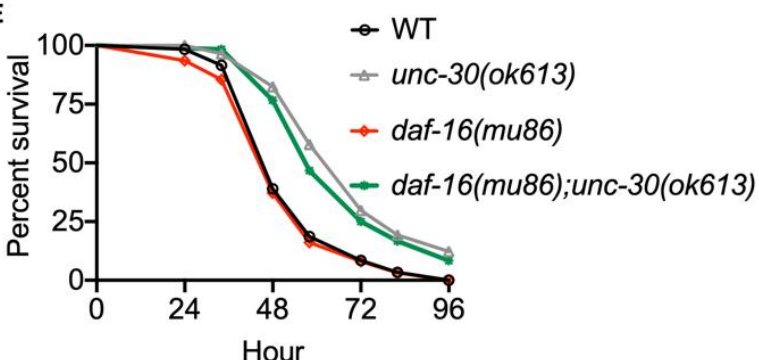

G

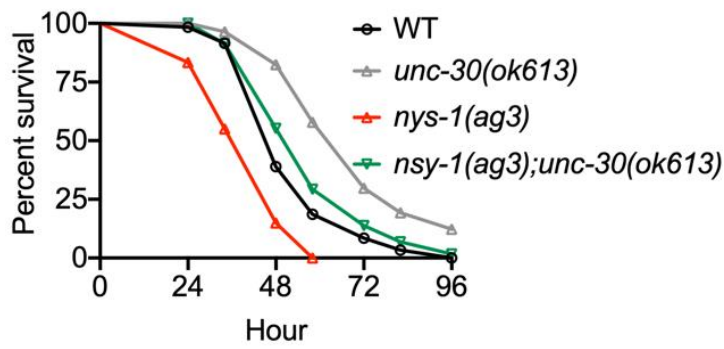

B
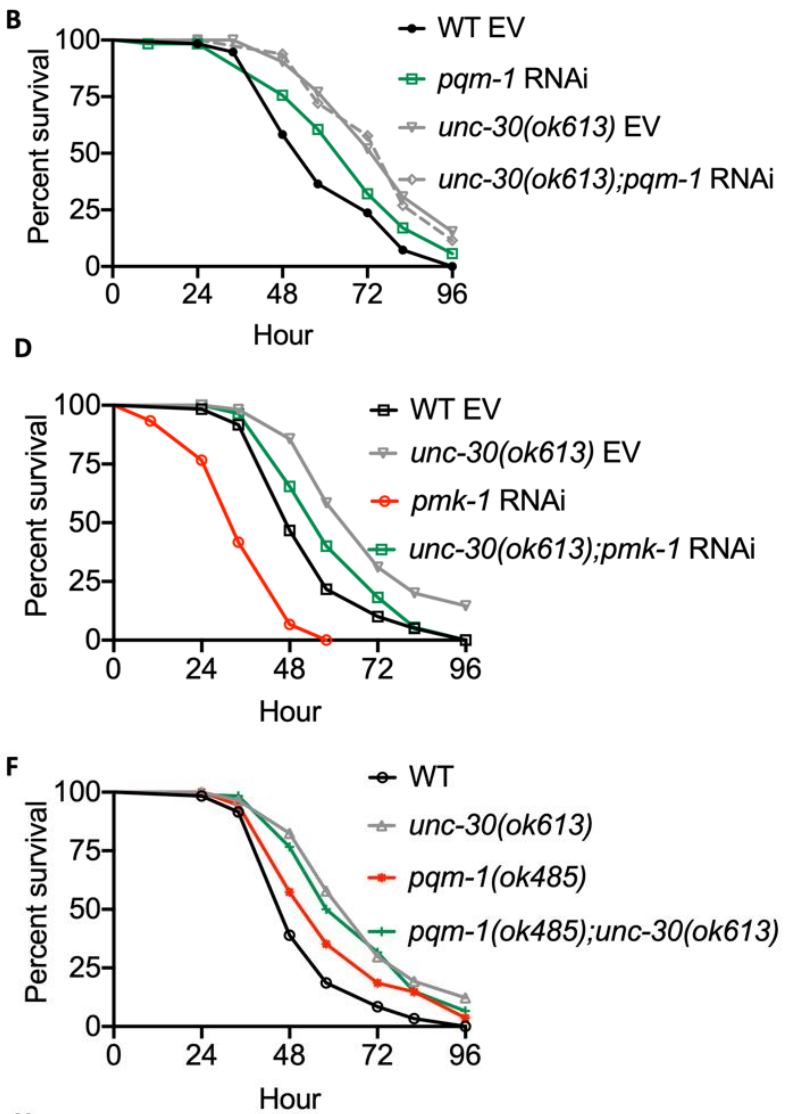

H

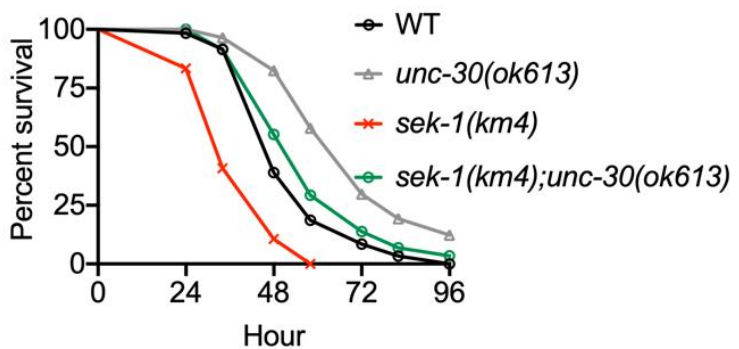

Figure 3. Functional loss of UNC-30 enhances immunity via the GATA/ELT-2 transcription factor and partly p38 MARK/PMK-1 pathway.

(A) WT, daf-16 RNAi, unc-30(ok613), and unc-30(ok613);daf-16 RNAi animals were exposed to $P$. aeruginosa and scored for survival. EV, empty vector RNAi control. WT EV vs. unc-30(ok613) EV, P < 0.0001; daf-16 RNAi, P =NS; unc-30(ok613);daf16 RNAi, $\mathrm{P}<0.0001$.

(B) WT, pqm-1 RNAi, unc-30(ok613), and unc-30(ok613);pqm-1 animals were exposed to $P$. aeruginosa and scored for survival. EV, empty vector RNAi control. WT EV vs. unc-30(ok613) EV, P < 0.0001; pqm-1 RNAi, P <0.05; unc-30(ok613);pqm-1 RNAi, $\mathrm{P}<0.0001$.

(C) WT, elt-2 RNAi, unc-30(ok613), and unc-30(ok613);elt-2 RNAi animals were exposed to $P$. aeruginosa and scored for survival. EV, empty vector RNAi control. WT EV vs. unc-30(ok613) EV, P < 0.0001; elt-2 RNAi, P <0.0001; unc30(ok613); elt-2 RNAi, $\mathrm{P}<0.0001$. elt-2 RNAi vs unc-30(ok613) elt-2 RNAi, $\mathrm{P}=\mathrm{NS}$.

(D) WT, pmk-1 RNAi, unc-30(ok613), and unc-30(ok613);pmk-1 RNAi animals were exposed to $P$. aeruginosa and scored for survival. EV, empty vector RNAi control. WT EV vs. unc-30(ok613) EV, P < 0.0001; pmk-1 RNAi, P <0.0001; unc- 
bioRxiv preprint doi: https://doi.org/10.1101/2021.02.25.432801; this version posted February 26, 2021. The copyright holder for this preprint (which was not certified by peer review) is the author/funder. All rights reserved. No reuse allowed without permission. 30(ok613);pmk-1 RNAi, P<0.05. pmk-1 RNAi vs unc-30(ok613) pmk-1 RNAi, $\mathrm{P}<0.0001$.

(E) WT, daf-16(mu86), unc-30(ok613), and daf-16(mu86);unc-30(ok613) animals were exposed to $P$. aeruginosa and scored for survival. WT vs. unc-30(ok613), $\mathrm{P}<0.0001$; daf-16(mu86), $\mathrm{P}=\mathrm{NS} ;$ daf-16(mu86);unc-30(ok613), $\mathrm{P}<0.0001$.

(F) WT, pqm-1(ok485), unc-30(ok613), and pqm-1(ok485);unc-30(ok613) animals were exposed to $P$. aeruginosa and scored for survival. WT vs. unc-30(ok613), $\mathrm{P}<0.0001$; pqm-1(ok485), $\mathrm{P}<0.05$; pqm-1(ok485);unc-30(ok613), $\mathrm{P}<0.0001$.

(G) WT, nsy-1(ag3), unc-30(ok613), and nsy-1(ag3);unc-30(ok613) animals were exposed to $P$. aeruginosa and scored for survival. WT vs. unc-30(ok613), $\mathrm{P}<0.0001$; nsy-1(ag3), $\mathrm{P}<0.0001$; nsy-1(ag3); unc-30(ok613), $\mathrm{P}=\mathrm{NS}$.

(H) WT, sek-1(km4), unc-30(ok613), and sek-1(km4);unc-30(ok613) animals were exposed to $P$. aeruginosa and scored for survival. WT vs. unc-30(ok613), $\mathrm{P}<0.0001$; sek-1(km4), $\mathrm{P}<0.0001$; sek-1(km4);unc-30(ok613), $\mathrm{P}=\mathrm{NS}$. 
bioRxiv preprint doi: https://doi.org/10.1101/2021.02.25.432801; this version posted February 26, 2021. The copyright holder for this preprint (which was not certified by peer review) is the author/funder. All rights reserved. No reuse allowed without permission.

A

Age-related genes

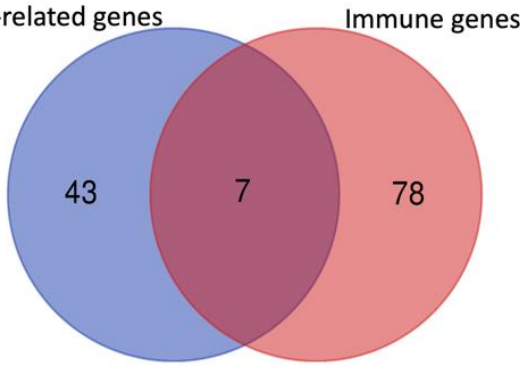

C

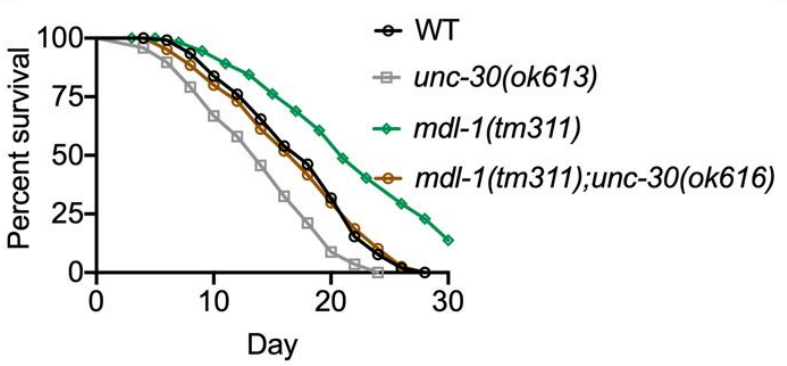

E

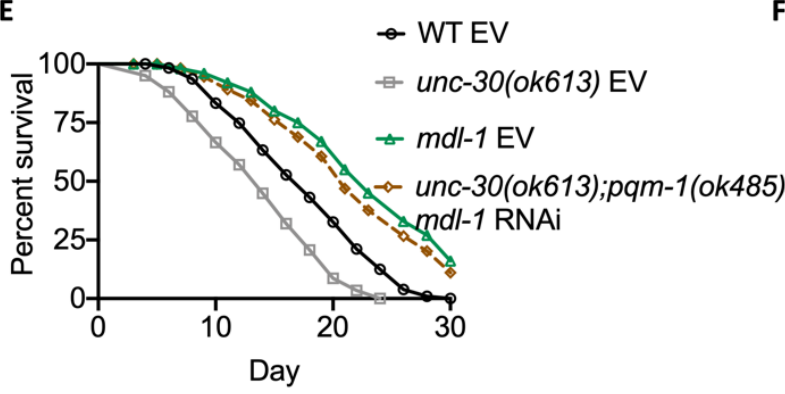

B

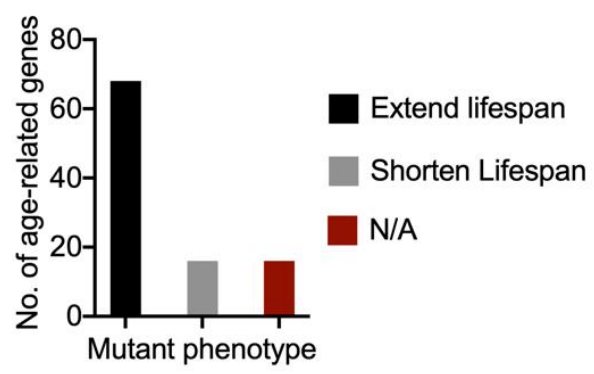

D
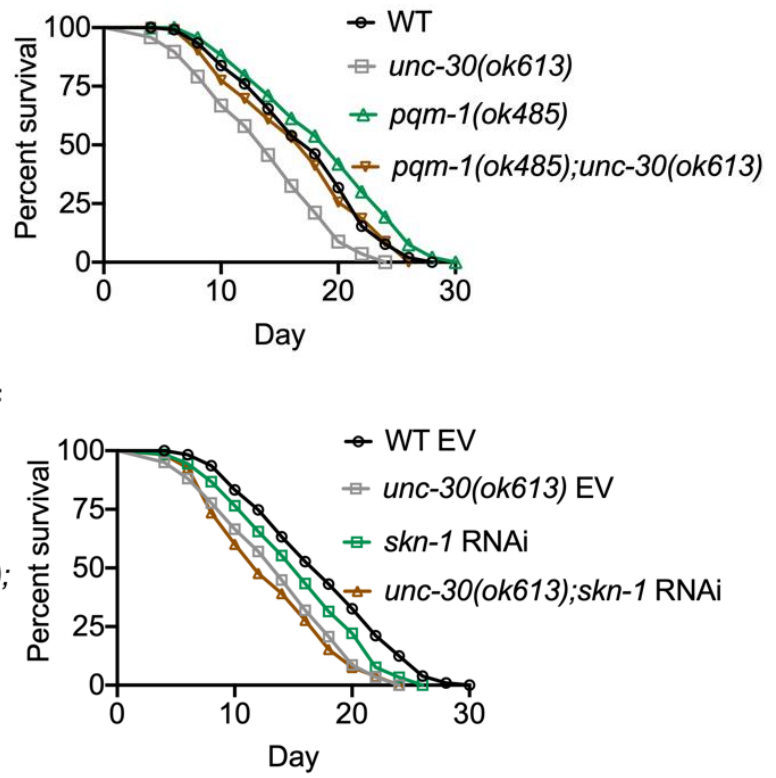

Figure 4. Functional loss of UNC-30 reduces longevity via MXD3/MDL-1 and PQM-1 pathways

(A) Venn diagram of the immune and aging genes upregulated in unc-30(ok613) vs WT non-infected animals.

(B) Number of age determination genes upregulated in unc-30(ok613) vs WT for which mutant phenotypes have been reported.

(C) WT, mdl-1(tm311), unc-30(ok613), and mdl-1(tm311);unc-30(ok613) animals were exposed to UV-killed E. coli and scored for survival. WT vs. unc-30(ok613), P < 0.0001; mdl-1(tm311), P < 0.0001; mdl-1(tm311);unc-30(ok613), P=NS. mdl1(tm311) vs $m d l-1($ tm311); unc-30(ok613), $\mathrm{P}<0.001$.

(D) WT, pqm-1(ok485), unc-30(ok613), and pqm-1(ok485);unc-30(ok613) animals were exposed to UV-killed E. coli and scored for survival. WT vs. unc-30(ok613), P < 0.0001; unc-30(ok613);pqm-1(ok485), $\mathrm{P}<0.0001$. unc-30(ok613) vs unc30(ok613);pqm-1(ok485), $\mathrm{P}<0.05$.

(E) WT, unc-30(ok613), and unc-30(ok613);pqm-1(ok485);mdl-1 RNAi animals were exposed to UV-killed $E$. coli and scored for survival. EV, empty vector RNAi control. WT EV vs. unc-30(ok613) EV, P < 0.0001; unc-30(ok613);pqm-1(ok485);mdl-1 RNAi, P<0.001. unc-30(ok613);pqm-1(ok485); mdl-1 RNAi vs. mdl-1 RNAi, P=NS.

(F) WT, skn-1 RNAi, unc-30(ok613), and unc-30(ok613);skn-1 RNAi animals were exposed to UV-killed $E$. coli and scored for survival. EV, empty vector RNAi control. WT EV vs unc-30(ok613) EV, P < 0.0001; skn-1(mg570)/skn-1 RNAi, P < 0.001; unc-30(ok613); skn-1 RNAi, P<0.0001. skn-1 RNAi vs unc-30(ok613);skn-1 RNAi, $\mathrm{P}<0.001$. 
bioRxiv preprint doi: https://doi.org/10.1101/2021.02.25.432801; this version posted February 26, 2021. The copyright holder for this preprint (which was not certified by peer review) is the author/funder. All rights reserved. No reuse allowed without permission.

A

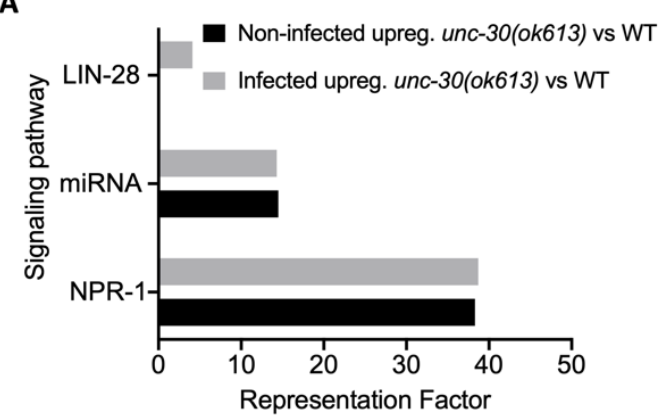

C

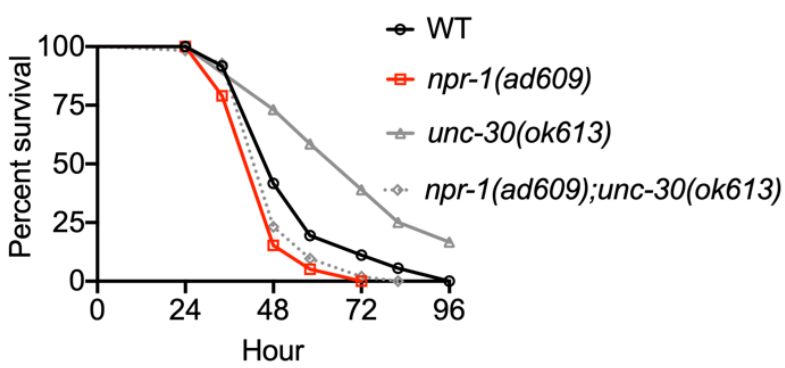

E

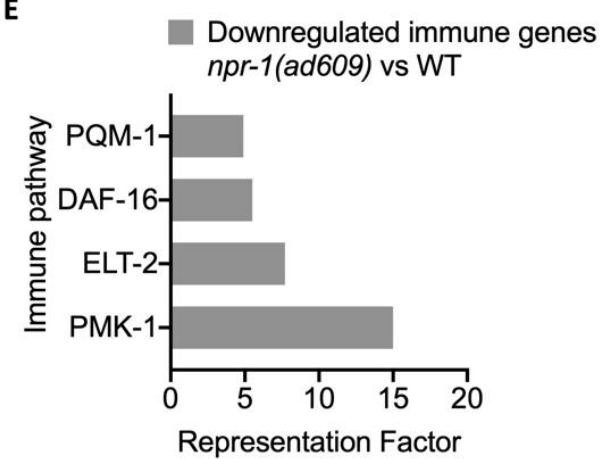

B

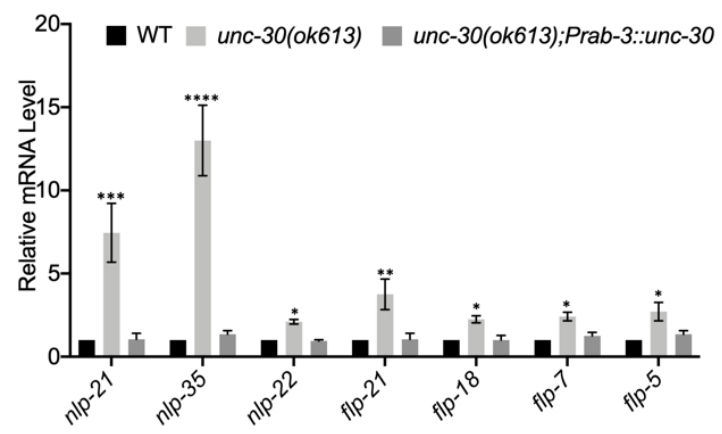

D

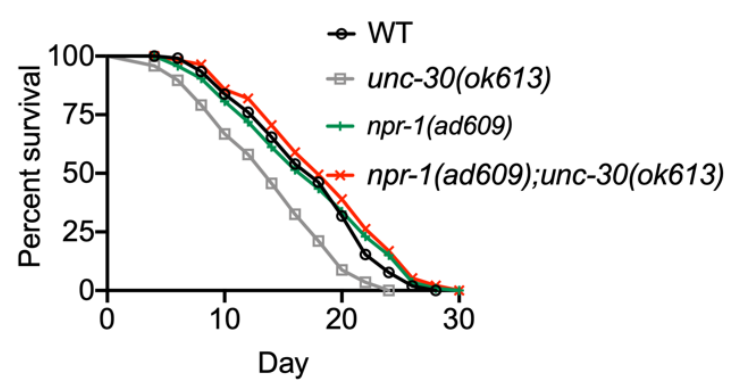

$\mathbf{F}$

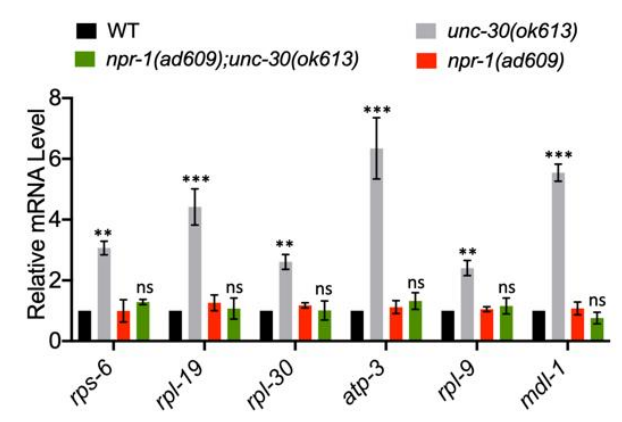

Figure 5. PITX1/UNC-30 regulates immunity and longevity via neuropeptide signaling.

(A) Representation factors of neuropeptide genes upregulated in non-infected and $P$. aeruginosa-infected unc-30(ok613) vs WT animals.

(B) qRT-PCR analysis of neuropeptide gene expression in WT, unc-30(ok613) and unc30(ok613);Prab-3::unc-30 animals. Bars represent means while error bars indicate $\mathrm{SD} ; * \mathrm{p}<0.05, * * \mathrm{p}<0.001$ and $* * * \mathrm{p}<0.0001$.

(C) WT, npr-1(ad609), unc-30(ok613), and npr-1(ad609);unc-30(ok613) animals were exposed to $P$. aeruginosa and scored for survival. WT vs unc-30(ok613), $\mathrm{P}<0.0001$; npr-1(ad609), $\mathrm{P}<0.001$; npr-1(ad609); unc-30(ok613), $\mathrm{P}<0.05$. npr-1(ad609) vs $n p r-$ 1(ad609)::unc-30(ok613), $\mathrm{P}=\mathrm{NS}$.

(D) WT, npr-1(ad609), unc-30(ok613), and unc-30(ok613);npr-1(ad609) animals were exposed to UV-killed E. coli and scored for survival. WT vs unc-30(ok613) $\mathrm{P}<$ 0.0001; npr-1(ad609), $\mathrm{P}=\mathrm{NS}$. While npr-1(ad609) vs unc-30(ok613), $\mathrm{P}<0.0001$; unc30(ok613); $n p r-1$ (ad609), $\mathrm{P}=\mathrm{NS}$.

(E) Representation factors of immune genes downregulated in $n p r-1$ (ad609) vs WT.

(F) qRT-PCR analysis of age determination gene expression in WT, npr-1(ad609), unc30(ok613), and unc-30(ok613);npr-1(ad609) animals animals. Bars represent means while error bars indicate SD; ${ }^{*} \mathrm{p}<0.05, * * \mathrm{p}<0.001$ and $* * * \mathrm{p}<0.0001$. 
A

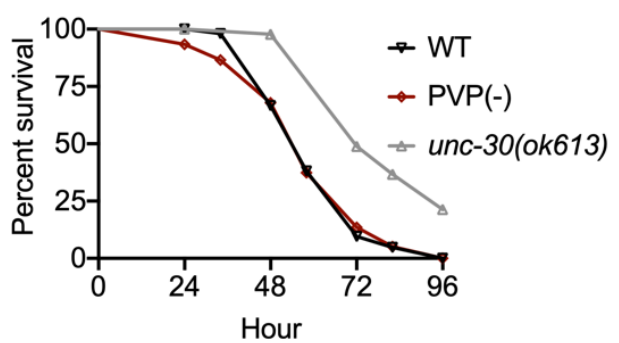

C
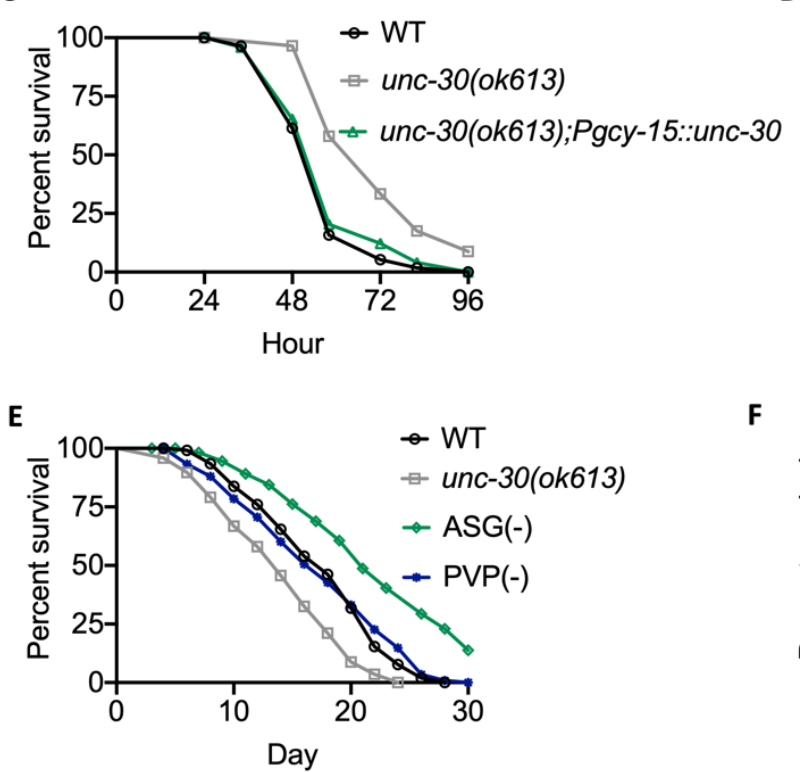

B

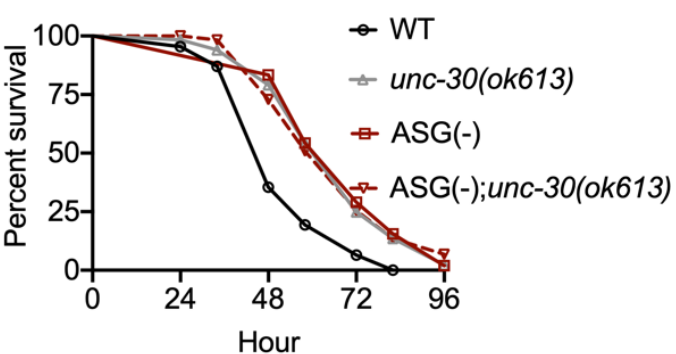

D

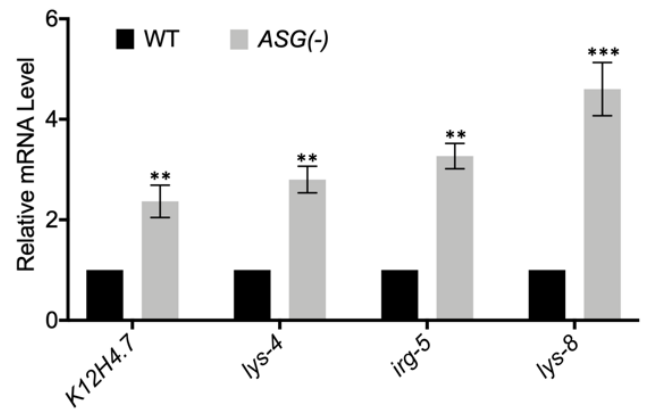

$\mathbf{F}$

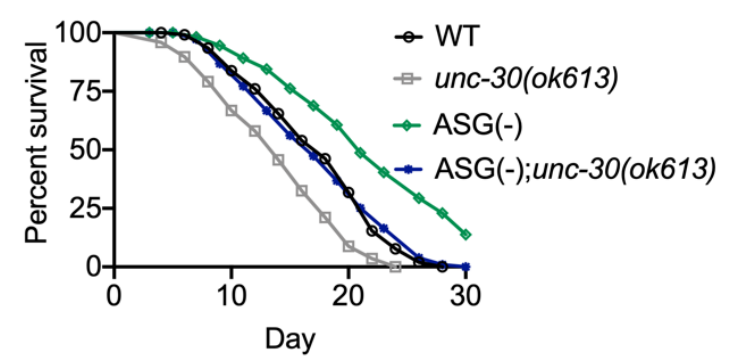

G

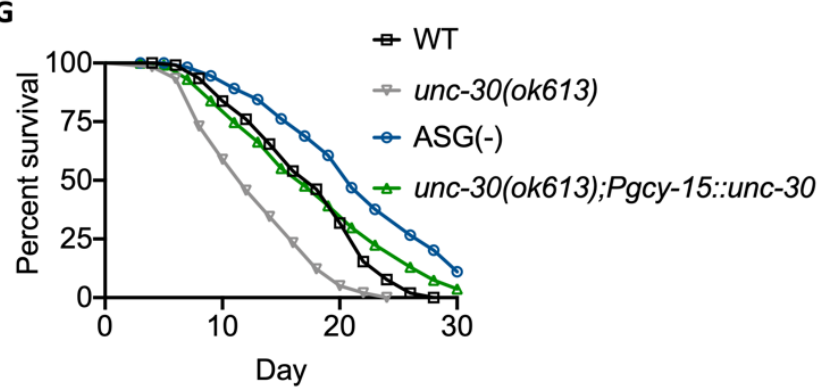

Figure 6. ASG neurons control the immunity-longevity tradeoff

(A) WT, PVP (-), and unc-30(ok613) animals were exposed to P. aeruginosa and scored for survival. WT vs PVP (-), P = NS.

(B) WT, unc-30(ok613), ASG(-), and ASG(-);unc-30(ok613) animals were exposed to $P$. aeruginosa and scored for survival. WT vs ASG(-), P < 0.0001; ASG(-); unc30(ok613), $\mathrm{P}<0.0001$. While ASG(-) vs. ASG(-);unc-30(ok613), $\mathrm{P}=\mathrm{NS}$.

(C) qRT-PCR analysis of immune gene expression in WT and ASG(-) animals. Bars represent means while error bars indicate SD; **p $<0.001$ and $* * * p<0.0001$.

(D) WT, unc-30(ok613), unc-30(ok613);Pgyc-15::unc-30 animals were exposed to $P$. aeruginosa and scored for survival. WT vs unc-30(ok613);Pgyc-15::unc-30, $\mathrm{P}=\mathrm{NS}$.

(E) WT, ASG(-), PVP(-), and unc-30(ok613) animals were exposed to UV-killed E. coli and scored for survival. WT vs ASG(-), P < 0.0001; unc-30(ok613), $\mathrm{P}<0.0001$. 
bioRxiv preprint doi: https://doi.org/10.1101/2021.02.25.432801; this version posted February 26, 2021. The copyright holder for this preprint (which was not certified by peer review) is the author/funder. All rights reserved. No reuse allowed without permission.

(F) WT, ASG(-), ASG(-);unc-30(ok613), and unc-30(ok613) animals were exposed to UV-killed E. coli and scored for survival. WT vs ASG(-), P <0.0001; unc-30(ok613), $\mathrm{P}<0.0001$. unc-30(ok613) vs ASG(-)::unc-30(ok613), $\mathrm{P}<0.001$.

(G) WT, unc-30(ok613), unc-30(ok613);Pgyc-15::unc-30 animals were exposed to UVkilled E. coli and scored for survival. WT vs unc-30(ok613);Pgyc-15::unc-30, $\mathrm{P}=$ NS.

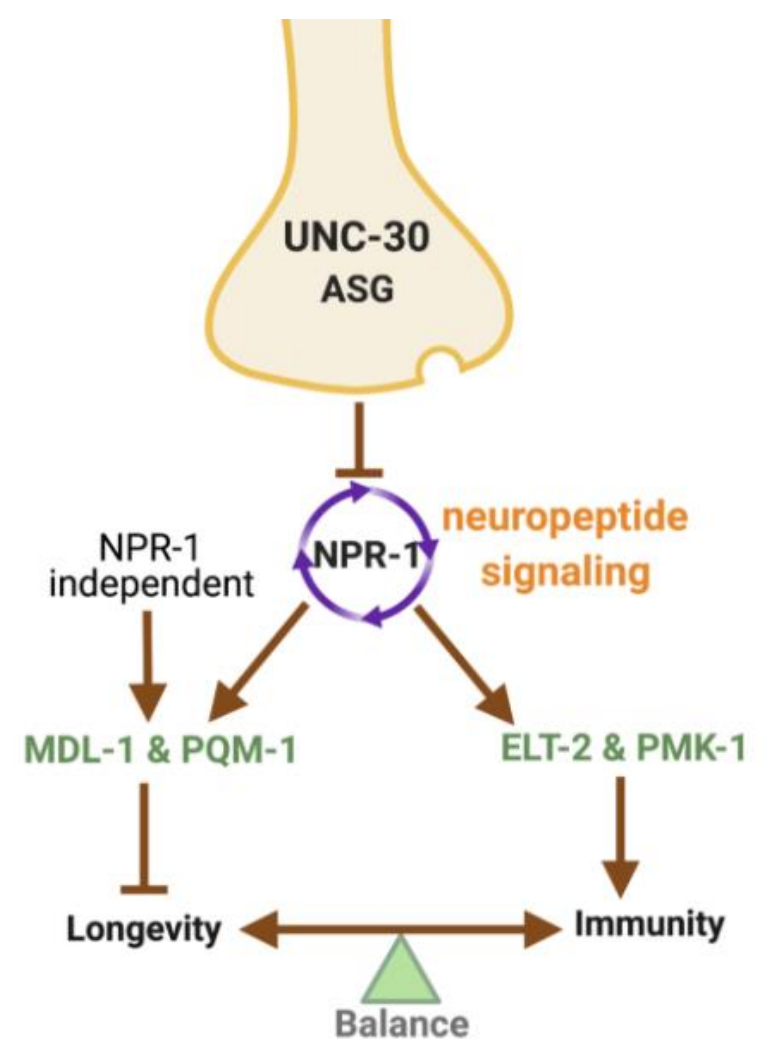

Figure 7. Model for neuronal control of the tradeoff between immunity and longevity via neuropeptide signaling in $\boldsymbol{C}$. elegans. Neuronal PITX1/UNC-30, via the NPR-1, inhibits immunity by preventing the expression of ELT-2- and PMK-1-dependent immune genes and promotes longevity by preventing the expression of MDL-1 and PQM-1-dependent agerelated genes. 